\title{
Groundwater Pathway Scoping Analysis for Disposal of APT and TEF Wastes
}

by

J. R. Cook

Westinghouse Savannah River Company

Savannah River Site

Aiken, South Carolina 29808

DOE Contract No. DE-AC09-96SR18500

This paper was prepared in connection with work done under the above contract number with the U.S. Department of Energy. By acceptance of this paper, the publisher and/or recipient acknowiedges the U.S. Government's right to retain a nonexclusive, royalty-free license in and to any copyright covering this paper, along with the right to reproduce and to authorize others to reproduce all or part of the copyrighted paper. 


\section{DISCLAIMER}

This report was prepared as an account of work sponsored by an agency of the United States Government. Neither the United States Government nor any agency thereof, nor any of their employees, makes any warranty, express or implied, or assumes any legal liability or responsibility for the accuracy, completeness, or usefulness of any information, apparatus, product, or process disclosed, or represents that its use would not infringe privately owned rights. Reference herein to any specific commercial product, process, or service by trade name, trademark, manufacturer, or otherwise does not necessarily constitute or imply its endorsement, recommendation, or favoring by the United States Government or any agency thereof. The views and opinions of authors expressed herein do not necessarily state or reflect those of the United States Government or any agency thereof.

This report has been reproduced directly from the best available copy.

- Available to DOE and DOE contractors from the Office of Scientific and Technical Information, P. O. Box 62, Oak Ridge, TN 37831; prices available from (423) 576-8401.

Available to the public from the National Technical Information Service, U. S. Department of Commerce, 5285 Port Royal Road, Springfield, VA 22161. 


\section{DISCLAIMER}

Portions of this document may be illegible in electronic image products. Images are produced from the best available original document. 
WESTINGHOUSE SAVANNAH RIVER COMPANY

SAVANNAH RIVER TECHNOLOGY CENTER
WSRC-RP-98-00729

Keywords: Performance Assessment Waste Management

Radioactive Waste

Retention: Permanent

GROUNDWATER PATHWAY SCOPING ANALYSIS FOR DISPOSAL OF APT AND TEF WASTES

Date of Report: August 20, 1998

Author:

James R. Cook, 773-43A 
GROUNDWATER PATHWAY SCOPING ANALYSIS FOR DISPOSAL OF APT AND REF WASTES
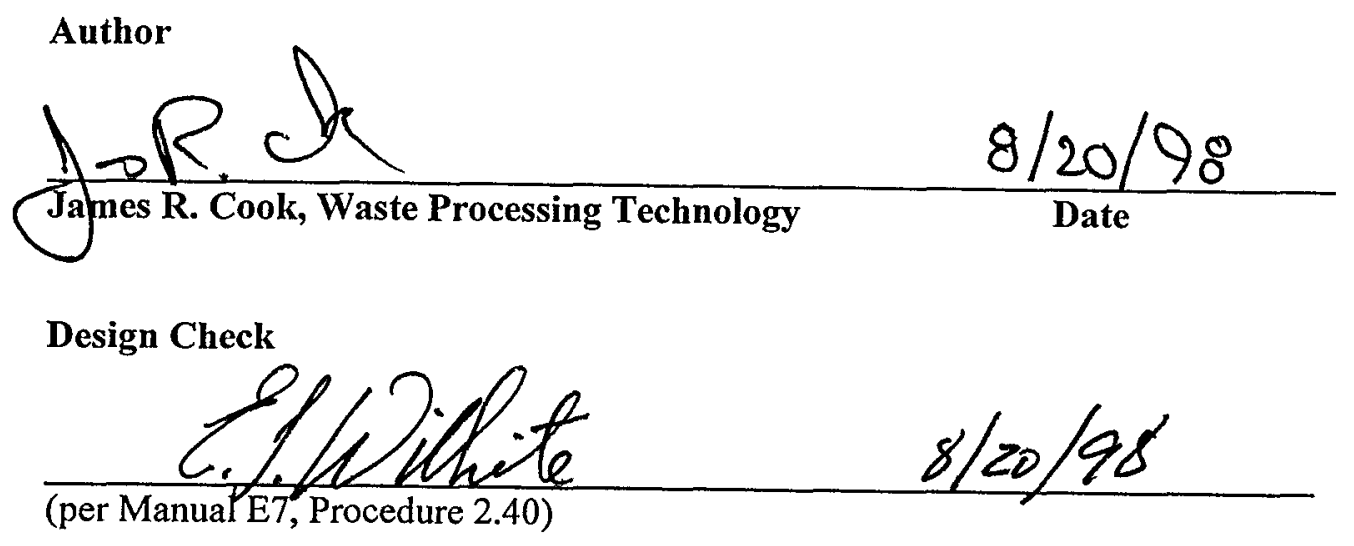

Approvals/Review

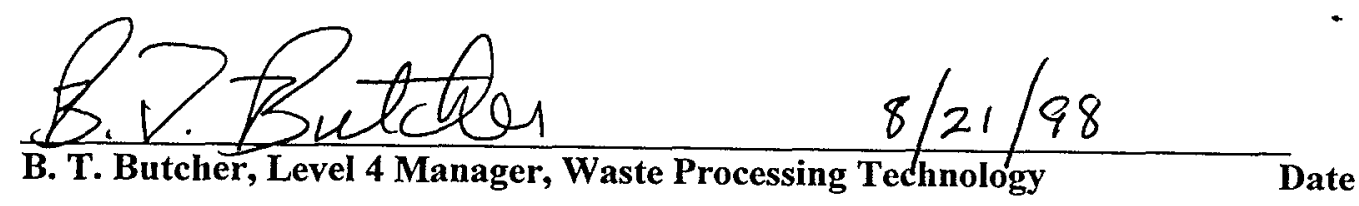




\section{GROUNDWATER PATHWAY SCOPING ANALYSIS OF APT AND TEF WASTES}

\section{Introduction and Summary}

The methods used to analyze the long-term performance of APT and TEF waste, with respect to the groundwater pathway, disposed in Intermediate Level vaults are described in this report. The purpose of this work is to reduce the number of radionuclides that need to be considered in a detailed analysis of performance. A methodology was selected that is more rigorous than the screening analysis described earlier ${ }^{1}$, but still conservative enough that radionuclides shown to have minimal dose impact using the methodology can be neglected with assurance that they would not have produced significant results if included in a detailed computer model.

The screening analysis started with 826 radionuclides and reduced the number to 56 that could potentially be of concern. The screening study used a methodology developed by the National Council on Radiation Protection and Measurements ${ }^{2}$ for releases of radionuclides to groundwater.

The PATHRAE computer code ${ }^{3}$ was used for this scoping study. The code is described in the following section. This is followed by a description of the conceptual model used and a discussion of the important parameter values used and their sources. Lastly the results of the study are presented.

This work further reduced the number of radionuclides needing detailed analysis from 56 to 23 .

\section{Description of the PATHRAE Code}

The purpose of the PATHRAE computer code ${ }^{3}$ is to calculate doses and health effects which might be caused by disposal of waste material in the near surface environment. The code was developed by Rogers and Associates Engineering Corporation, of Salt Lake City, Utah, for the U.S. Environmental Protection Agency, and is accepted by that organization. PATHRAE was selected for use in the Environmental Impact Statement on Waste Management Activities and Groundwater Protection at the Savannah River Site. 
Input to the code consists of a number of parameters which describe the waste and the characteristics of the disposal site and the surrounding area. The more that is known about the waste and the site, the more confidence there will be in the results produced by the code.

The PATHRAE code can calculate doses due to a number of exposure pathways: groundwater transport to a surface stream, groundwater use in a nearby well, surface erosion and subsequent waste exposure, trench overflow (bathtub effect), food grown on the site, biointrusion into the waste, direct gamma exposure, inhalation of dust on-site, inhalation of radon gas, and atmospheric transport of particulates offsite.

The code assumes that the waste inventory is evenly distributed throughout the waste volume. For the groundwater transport pathways a specified fraction of the inventory is leached from the waste each year and transported vertically through the unsaturated zone, and then horizontally to hypothetical wells at locations at 1 meter, 100 meters, and to a nearby stream. The water velocity through the unsaturated zone is calculated in the code from input values for infiltration, soil porosity and saturated hydraulic conductivity. The average linear velocity in the water table aquifer is a code input, the value of which is taken from three-dimensional numerical modeling work performed for the E- Area Vault Performance Assessment ${ }^{5}$.

The velocity of each radionuclide considered is calculated based on the partition coefficient (commonly called $\mathrm{Kd}$, and referred to as aquifer sorption in PATHRAE) and the water velocity. A quantity called the retardation factor is calculated from the partition coefficient and used to modify the velocity of a species relative to the water velocity.

The Erosion pathway considers the effects of erosion of the cover material and transport and deposition in a nearby stream.

The pathway called the Bathtub Effect calculates doses due to disposal trenches overflowing if geologic and precipitation conditions are such that this phenomenon might occur.

The Food Grown on Site pathway assumes that at some time in the future someone moves onto the disposal facility and constructs a home with a basement and a water well. Excavation of the basement and well construction brings some portion of the buried waste to the surface, which is then used to grow food in a small garden. The person is thus exposed to radiation from the food he eats. 
The Biointrusion pathway is essentially the same as the Food Grown on site except that no home is constructed.

The Direct Gamma pathway considers the effects of penetrating gamma radiation from the buried waste both to workers during the operational phase and to someone occupying the disposal site and disturbing the waste sometime in the future.

The Dust pathway considers inhalation of resuspended dust by an intruder at the waste disposal facility due to house construction and well drilling.

The Radon Inhalation pathway calculates doses due to radon in-growth and radon daughters in a structure built over a disposal facility.

The Atmospheric Transport pathway considers off-site transport of materials due to dust resuspension, incineration, or trench fire.

In each of these pathways a hypothetical person is exposed to radiation released from the disposal facility. The PATHRAE code calculates the dilution, dispersion, or attenuation provided by the waste site and the environment, and thus the curie concentration of each radionuclide to which the person will be exposed. For the ingestion and inhalation exposure pathways, dose conversion factors from the U. S. Department of Energy ${ }^{6}$ are used to calculate annual doses from curie concentrations. Dose conversion factors for direct gamma exposure are taken from the PRESTO data base?

As stated above, the PATHRAE code was used as the basis for dose calculations for the Groundwater Protection and Waste Management Environmental Impact Statement issued by DOE in 1987. One of the supporting documents for the EIS was a quality assurance report on the models used. ${ }^{8}$ Several levels of review are documented in this report, (1) review of code documentation, history of use, and previous validation and verification studies, (2) comparison of model results to alternate models using different boundary conditions, (3) comparison of model predictions to measured concentrations, and (4) sensitivity analysis to identify critical input parameters.

In this work, only the Groundwater to a Well pathway was used. 


\section{Conceptual Model}

For the purpose of scoping, the entire 40 year projected inventory of both APT and TEF waste was assumed to be disposed in one Intermediate Level vault measuring $85 \mathrm{~m}$ long, $15 \mathrm{~m}$ wide and $8 \mathrm{~m}$ high. This inventory is shown in Table 1. Vault and site characteristics were taken from the EAV PA. ${ }^{4}$

A study of corrosion rate of both the wasteforms and the steel boxes ${ }^{9}$ was used to estimate the time of release and the release rate into the Intermediate Level Vault. All of the inventory was assumed to be in one waste form. A corrosion rate of 2.5 microns/yr was assumed for the wasteform. Assuming that contamination is distributed evenly throughout the assumed $1 \mathrm{~cm}$ thickness of the wasteform, the contaminant release fraction is $2 . \times 10^{-4}$ per year.

The corrosion rate given for the waste containers was $1 \times 10^{-5} \mathrm{~m} / \mathrm{yr}$. The containers are $1 / 2$ inch thick. Assuming the containers will fail when they have corroded to one-half of their original thickness, the time of failure will be 635 years. This length of time is greater than the intact period of the Intermediate Level Vault ( 575 years). Therefore, for the scoping model it was assumed that no release or corrosion occurred in the first 635 years after disposal.

Partition coefficient values for the elements included in the scoping analysis and the source of the values are given in Table 2 .

All parameters for the PATHRAE code were taken from values used in the EAV Performance Assessment for new radioactive waste disposal ${ }^{4}$. The parameters used can be found in Attachment 1 , the output from the PATHRAE program.

\section{Results}

The peak concentrations, doses and the years of the peak occurrences are given in Table 3. Those radionuclides that had a peak dose greater that $1 \mathrm{mrem} /$ year were selected for inclusion in the detailed model. These are shown in Table 4. 
Table 1. Inventory used in scoping analysis

\begin{tabular}{|c|c|c|c|c|c|c|c|c|}
\hline & $\mathrm{APT}$ & APT & $\mathrm{APT}$ & APT & APT & TEF & TEF & Totals \\
\hline & Tungston & Window & Steel & Aluminum & Lead & TPBARS & Baseplate & \\
\hline & $\overline{\mathrm{Ci}}$ & $\mathrm{Ci}$ & $\mathrm{Ci}$ & $\mathrm{Ci}$ & $\mathrm{Ci}$ & $\mathrm{Ci}$ & $\mathrm{Ci}$ & $\mathrm{Ci}$ \\
\hline$\overline{\mathrm{H}-3}$ & $2.1 \mathrm{E}+08$ & $2.7 \mathrm{E}+07$ & $5.4 \mathrm{E}+07$ & $9.2 \mathrm{E}+06$ & $8.8 \mathrm{E}+07$ & $1.9 \mathrm{E}+09$ & & $2.3 \mathrm{E}+09$ \\
\hline$\overline{\mathrm{Be}-10}$ & $1.8 \mathrm{E}+00$ & $3.7 \mathrm{E}-01$ & $7.4 \mathrm{E}-01$ & $1.4 \mathrm{E}+00$ & $5.8 \mathrm{E}+00$ & & & $1.0 \mathrm{E}+01$ \\
\hline $\mathrm{C}-14$ & $2.5 \mathrm{E}+03$ & $2.1 \mathrm{E}+02$ & $4.1 \mathrm{E}+02$ & $4.1 \mathrm{E}+02$ & $8.5 \mathrm{E}+03$ & $1.5 \mathrm{E}+03$ & $3.8 \mathrm{E}+03$ & $1.7 \mathrm{E}+04$ \\
\hline $\mathrm{Na}-22$ & $1.1 \mathrm{E}+05$ & $1.7 \mathrm{E}+06$ & $3.4 \mathrm{E}+06$ & $1.1 \mathrm{E}+07$ & $5.6 \mathrm{E}+06$ & & & $2.2 \mathrm{E}+07$ \\
\hline Al-26 & $1.6 \mathrm{E}+00$ & $3.2 \mathrm{E}+01$ & $6.4 \mathrm{E}+01$ & $7.1 \mathrm{E}+02$ & $4.5 \mathrm{E}+02$ & & & $1.3 \mathrm{E}+03$ \\
\hline Si-32 & $1.4 \mathrm{E}+00$ & 6.4E-01 & $1.3 \mathrm{E}+00$ & $6.3 \mathrm{E}-05$ & \begin{tabular}{|c|}
$5.0 \mathrm{E}-02$ \\
\end{tabular} & & & $3.4 \mathrm{E}+00$ \\
\hline $\mathrm{Cl}-36$ & $2.3 \mathrm{E}+01$ & $1.6 \mathrm{E}+02$ & $3.1 \mathrm{E}+02$ & $8.1 \mathrm{E}-10$ & $1.2 \mathrm{E}+01$ & & & $5.1 \mathrm{E}+02$ \\
\hline $\mathrm{K}-40$ & $5.7 \mathrm{E}-03$ & $3.4 \mathrm{E}-02$ & $6.8 \mathrm{E}-02$ & $1.6 \mathrm{E}-08$ & $2.8 \mathrm{E}-02$ & & & $1.4 \mathrm{E}-01$ \\
\hline Ca-41 & $7.1 \mathrm{E}+01$ & $3.1 \mathrm{E}+02$ & $6.2 \mathrm{E}+02$ & $7.3 \mathrm{E}+00$ & $3.0 \mathrm{E}+02$ & & & $1.3 \mathrm{E}+03$ \\
\hline Ti-44 & $1.6 \mathrm{E}+04$ & $1.3 \mathrm{E}+05$ & $2.5 \mathrm{E}+05$ & $1.1 \mathrm{E}-09$ & & & & $4.0 \mathrm{E}+05$ \\
\hline V-49 & $2.9 \mathrm{E}+05$ & $1.8 \mathrm{E}+06$ & $3.7 \mathrm{E}+06$ & $7.4 \mathrm{E}+04$ & $1.1 \mathrm{E}+05$ & & & $6.0 \mathrm{E}+06$ \\
\hline Mn-53 & $1.5 \mathrm{E}+01$ & $2.0 \mathrm{E}+01$ & $4.0 \mathrm{E}+01$ & $2.1 \mathrm{E}-09$ & $3.4 \mathrm{E}-01$ & & & $7.5 \mathrm{E}+01$ \\
\hline Fe-60 & $1.9 \mathrm{E}-01$ & $4.0 \mathrm{E}-01$ & $7.9 \mathrm{E}-01$ & $6.2 \mathrm{E}-06$ & 7.3E-01 & & & $2.1 \mathrm{E}+00$ \\
\hline $\mathrm{Ni}-59$ & $2.5 \mathrm{E}+02$ & $1.0 \mathrm{E}+03$ & $2.0 \mathrm{E}+03$ & $6.2 \mathrm{E}-06$ & $1.9 \mathrm{E}-02$ & $3.7 \mathrm{E}+03$ & $1.7 \mathrm{E}+04$ & $2.4 \mathrm{E}+04$ \\
\hline $\mathrm{Ge}-68$ & $1.2 \mathrm{E}+03$ & $5.3 \mathrm{E}+03$ & $1.1 \mathrm{E}+04$ & & $3.8 \mathrm{E}+04$ & & & $5.6 \mathrm{E}+04$ \\
\hline Se-79 & $1.7 \mathrm{E}+01$ & $2.0 \mathrm{E}-03$ & $4.0 \mathrm{E}-03$ & & $8.9 \mathrm{E}+01$ & & & $1.1 \mathrm{E}+02$ \\
\hline Sr-90 & $8.5 \mathrm{E}+03$ & $4.1 \mathrm{E}-02$ & $8.2 \mathrm{E}-02$ & & $1.1 \mathrm{E}+05$ & & & $1.2 \mathrm{E}+05$ \\
\hline $\mathrm{Zr}-93$ & $8.2 \mathrm{E}-01$ & $5.6 \mathrm{E}-01$ & $1.1 \mathrm{E}+00$ & & $3.0 \mathrm{E}+00$ & $1.8 \mathrm{E}+01$ & & $2.3 \mathrm{E}+01$ \\
\hline$\overline{\mathrm{Nb}}-94$ & $2.7 \mathrm{E}+01$ & $3.7 \mathrm{E}+02$ & $7.5 \mathrm{E}+02$ & & $1.1 \mathrm{E}+02$ & $6.1 \mathrm{E}+01$ & $4.0 \mathrm{E}+01$ & $1.4 \mathrm{E}+03$ \\
\hline Mo-93 & $1.8 \mathrm{E}+00$ & $4.4 \mathrm{E}+02$ & $8.7 \mathrm{E}+02$ & & $7.7 \mathrm{E}+02$ & $1.2 \mathrm{E}+02$ & & $2.2 \mathrm{E}+03$ \\
\hline Tc-97 & $6.1 \mathrm{E}-01$ & $1.7 \mathrm{E}-06$ & $\begin{array}{ll}3.4 \mathrm{E}-06 \\
\end{array}$ & & $9.1 \mathrm{E}-01$ & & & $1.5 \mathrm{E}+00$ \\
\hline Tc-98 & $1.4 \mathrm{E}-01$ & $2.8 \mathrm{E}-05$ & $5.6 \mathrm{E}-05$ & & $3.4 \mathrm{E}-01$ & & & $4.8 \mathrm{E}-01$ \\
\hline Tc-99 & $5.4 \mathrm{E}+00$ & $4.8 \mathrm{E}+00$ & $9.7 \mathrm{E}+00$ & & $3.2 \mathrm{E}+01$ & $3.1 \mathrm{E}+01$ & $1.0 \mathrm{E}+01$ & $9.3 \mathrm{E}+01$ \\
\hline Rh-101 & $2.2 \mathrm{E}+05$ & & & & $5.8 \mathrm{E}+05$ & & & $8.0 \mathrm{E}+05$ \\
\hline Rh-102m & $1.2 \mathrm{E}+02$ & & & & $9.3 \mathrm{E}+03$ & & & $9.4 \mathrm{E}+03$ \\
\hline Rh-102 & $6.2 \mathrm{E}+04$ & $2.2 \mathrm{E}-09$ & $4.4 \mathrm{E}-09$ & & $1.3 \mathrm{E}+05$ & & & $1.9 \mathrm{E}+05$ \\
\hline $\mathrm{Ag}-108 \mathrm{~m}$ & $2.2 \mathrm{E}+01$ & & & & $1.3 \mathrm{E}+01$ & & & $3.5 \mathrm{E}+01$ \\
\hline Sb-126 & & & & & $1.2 \mathrm{E}-05$ & & & $1.2 \mathrm{E}-05$ \\
\hline $\mathrm{I}-129$ & $1.8 \mathrm{E}-02$ & & & & $3.3 \mathrm{E}-02$ & & & $5.0 \mathrm{E}-02$ \\
\hline Cs-135 & $9.0 \mathrm{E}-03$ & & & & $2.7 \mathrm{E}-01$ & & & $2.8 \mathrm{E}-01$ \\
\hline Cs-137 & $5.4 \mathrm{E}-02$ & & & & $1.2 \mathrm{E}+01$ & & & $1.2 \mathrm{E}+01$ \\
\hline $\mathrm{Ba}-133$ & $3.8 \mathrm{E}+06$ & & & & $1.4 \mathrm{E}+05$ & & & $3.9 \mathrm{E}+06$ \\
\hline La-137 & $1.4 \mathrm{E}+03$ & & & & $1.3 \mathrm{E}+01$ & & & $1.4 \mathrm{E}+03$ \\
\hline La-138 & $6.5 \mathrm{E}-05$ & & & & $6.9 \mathrm{E}-06$ & & & 7.2E-05 \\
\hline Pm-145 & $5.2 \mathrm{E}+06$ & & & & $4.8 \mathrm{E}+04$ & & & $5.2 \mathrm{E}+06$ \\
\hline Sm-146 & $1.4 \mathrm{E}+00$ & & & & $3.0 \mathrm{E}-02$ & & & $1.4 \mathrm{E}+00$ \\
\hline Sm-147 & $8.9 \mathrm{E}-04$ & & & & $1.5 \mathrm{E}-05$ & & & $9.1 \mathrm{E}-04$ \\
\hline Sm-151 & $3.8 \mathrm{E}+04$ & & & & $5.0 \mathrm{E}+03$ & & & $4.3 \mathrm{E}+04$ \\
\hline Eu-150 & $1.9 E+05$ & & & & $8.5 \mathrm{E}+03$ & & & $2.0 \mathrm{E}+05$ \\
\hline Eu-152 & $1.3 \mathrm{E}+06$ & & & & $9.7 \mathrm{E}+03$ & & & $1.3 \mathrm{E}+06$ \\
\hline Eu-154 & $1.3 \mathrm{E}+06$ & & & & $4.7 \mathrm{E}+04$ & & & $1.3 \mathrm{E}+06$ \\
\hline$\overline{G d}-148$ & $1.4 \mathrm{E}+06$ & & & & $4.2 \mathrm{E}+04$ & & & $1.4 \mathrm{E}+06$ \\
\hline
\end{tabular}


Table 1. Inventory used in scoping analysis (continued)

\begin{tabular}{|l|c|c|c|c|c|c|c|c|}
\hline & APT & APT & APT & APT & APT & TEF & TEF & Totals \\
\hline & Tungston & Window & Steel & Aluminum & Lead & TPBARS & Baseplate & \\
\hline & $\mathrm{Ci}$ & $\mathrm{Ci}$ & $\mathrm{Ci}$ & $\mathrm{Ci}$ & $\mathrm{Ci}$ & $\mathrm{Ci}$ & $\mathrm{Ci}$ & $\mathrm{Ci}$ \\
\hline $\mathrm{Tb}-157$ & $6.4 \mathrm{E}+05$ & & & & $2.0 \mathrm{E}+04$ & & & $6.6 \mathrm{E}+05$ \\
\hline $\mathrm{Tb}-158$ & $3.4 \mathrm{E}+05$ & & & & $1.8 \mathrm{E}+04$ & & & $3.6 \mathrm{E}+05$ \\
\hline $\mathrm{Ho}-166 \mathrm{~m}$ & $1.6 \mathrm{E}+03$ & & & & $3.3 \mathrm{E}+02$ & & & $1.9 \mathrm{E}+03$ \\
\hline $\mathrm{Hf}-178 \mathrm{~m}$ & $4.1 \mathrm{E}+02$ & & & & $4.2 \mathrm{E}+00$ & & & $4.1 \mathrm{E}+02$ \\
\hline $\mathrm{Hf}-182$ & $2.8 \mathrm{E}+00$ & & & & $7.0 \mathrm{E}-04$ & & & $2.8 \mathrm{E}+00$ \\
\hline $\mathrm{W}-181$ & $4.6 \mathrm{E}+04$ & & & & $8.5 \mathrm{E}+05$ & & & $9.0 \mathrm{E}+05$ \\
\hline $\mathrm{Pt}-193$ & $3.1 \mathrm{E}+02$ & & & & $4.1 \mathrm{E}+02$ & & & $7.2 \mathrm{E}+02$ \\
\hline $\mathrm{Tl}-204$ & $2.8 \mathrm{E}+01$ & & & & $4.1 \mathrm{E}+06$ & & & $4.1 \mathrm{E}+06$ \\
\hline $\mathrm{Pb}-202$ & & & & & $5.7 \mathrm{E}+07$ & & & $5.7 \mathrm{E}+07$ \\
\hline $\mathrm{Pb}-205$ & & & & & $1.7 \mathrm{E}+04$ & & & $1.7 \mathrm{E}+04$ \\
\hline $\mathrm{Bi}-207$ & & & & & $2.3 \mathrm{E}+02$ & & & $2.3 \mathrm{E}+02$ \\
\hline
\end{tabular}


Table 2. $K_{d}$ values used in scoping study

\begin{tabular}{|c|c|c|}
\hline Radionuclide & $\mathrm{K}_{\mathrm{d}}(\mathrm{mL} / \mathrm{g})$ & Reference \\
\hline$\overline{\mathrm{Ag}}$ & 90 & 10 \\
\hline$\overline{\mathrm{Al}}$ & 1500 & 11 \\
\hline $\mathrm{Ar}$ & 0 & assumed value for inert gas \\
\hline$\overline{\mathrm{Ba}}$ & 52 & 12 \\
\hline$\overline{\mathrm{Be}}$ & 250 & 10 \\
\hline$\overline{\mathrm{Bi}}$ & 100 & 10 \\
\hline$\overline{\mathrm{C}}$ & 2 & 13 \\
\hline$\overline{\mathrm{Ca}}$ & 5 & 10 \\
\hline $\mathrm{Cd}$ & 80 & 10 \\
\hline$\overline{\mathrm{Cl}}$ & 1.7 & 12 \\
\hline Co & 60 & 10 \\
\hline Cs & 330 & 15 \\
\hline$\overline{\mathrm{Eu}}$ & 240 & 10 \\
\hline $\mathrm{Fe}$ & 220 & 10 \\
\hline$\overline{\mathrm{Gd}}$ & 240 & 12 \\
\hline $\mathrm{Ge}$ & 130 & assumed same as Sn \\
\hline$\overline{\mathrm{H}}$ & 0 & 10 \\
\hline$\overline{\mathrm{Hf}}$ & 450 & 10 \\
\hline$\overline{\mathrm{Ho}}$ & 250 & 10 \\
\hline$\overline{\mathrm{I}}$ & 0.6 & $\overline{14}$ \\
\hline$\overline{\mathrm{K}}$ & 15 & 10 \\
\hline $\mathrm{Kr}$ & 0 & assumed value for inert gas \\
\hline $\mathrm{La}$ & 1200 & $\overline{12}$ \\
\hline$\overline{\mathrm{Mn}}$ & 50 & 10 \\
\hline$\overline{\mathrm{Mo}}$ & 10 & 10 \\
\hline $\mathrm{Na}$ & 76 & 12 \\
\hline $\mathrm{Nb}$ & 160 & 10 \\
\hline$\overline{\mathrm{Ni}}$ & 400 & 10 \\
\hline$\overline{\mathrm{Pb}}$ & 270 & 10 \\
\hline $\mathrm{Pm}$ & 240 & 12 \\
\hline$\overline{\mathrm{Pt}}$ & 30 & assumed same as $\mathrm{Au}$ \\
\hline$\overline{\mathrm{Rh}}$ & 52 & $\overline{12}$ \\
\hline $\mathrm{Se}$ & 150 & 10 \\
\hline$\overline{\mathrm{Si}}$ & 35 & 10 \\
\hline$\overline{\mathrm{Sm}}$ & 245 & 10 \\
\hline $\mathrm{Sn}$ & 130 & 10 \\
\hline$\overline{\mathrm{Sr}}$ & 10 & 14 \\
\hline $\mathrm{Tb}$ & 240 & 12 \\
\hline $\mathrm{Tc}$ & 0.36 & 14 \\
\hline $\mathrm{Tl}$ & 390 & 12 \\
\hline$\overline{\mathrm{Ti}}$ & 600 & assumed same as $\mathrm{Zr}$ \\
\hline$\overline{\mathrm{V}}$ & 160 & assumed same as $\mathrm{Nb}$ \\
\hline$\overline{\mathrm{W}}$ & 100 & $\overline{12}$ \\
\hline $\mathrm{Zr}$ & 600 & 10 \\
\hline
\end{tabular}


Table 3. Results of Scoping Analysis at the 1 meter well

\begin{tabular}{|c|c|c|c|}
\hline Radionuclide & Year of Peak & $\begin{array}{c}\text { Peak Concentration } \\
\mathrm{pCi} / \mathrm{L}\end{array}$ & $\begin{array}{l}\text { Peak Dose } \\
\text { (mrem/yr) }\end{array}$ \\
\hline $\mathrm{Ag}-108 \mathrm{~m}$ & 1590 & $1.89 \times 10^{-1}$ & $1.03 \times 10^{-3}$ \\
\hline Al-26 & 17714.9 & $4.73 \times 10^{4}$ & $2.73 \times 10^{2}$ \\
\hline Ba-133 & 1088.7 & $1.25 \times 10^{-24}$ & $2.92 \times 10^{-2 T}$ \\
\hline $\mathrm{Be}-10$ & 7020.2 & $2.22 \times 10^{3}$ & 6.82 \\
\hline $\mathrm{Bi}-207$ & 1506.8 & $1.38 \times 10^{-7}$ & $4.94 \times 10^{-10}$ \\
\hline $\mathrm{C}-14$ & 735.6 & $8.23 \times 10^{6}$ & $1.26 \times 10^{4}$ \\
\hline $\mathrm{Ca}-41$ & 875.8 & $6.84 \times 10^{5}$ & $5.99 \times 10^{2}$ \\
\hline $\mathrm{Cl}-36$ & 721.9 & $2.69 \times 10^{3}$ & $5.90 \times 10^{2}$ \\
\hline Cs-135 & 7552.9 & $4.73 \times 10^{1}$ & $2.45 \times 10^{-1}$ \\
\hline Cs-137 & 3508 & $6.64 \times 10^{-34}$ & $2.42 \times 10^{-35}$ \\
\hline Fe-60 & 8114.2 & $5.23 \times 10^{2}$ & $5.73 \times 10^{1}$ \\
\hline Gd-148 & 2726 & $1.25 \times 10^{-3}$ & $1.92 \times 10^{-4}$ \\
\hline $\mathrm{H}-3$ & .643 .4 & $2.18 \times 10^{-4}$ & $1.00 \times 10^{-8}$ \\
\hline $\mathrm{Hf}-182^{\circ}$ & 20441.7 & $3.51 \times 10^{2}$ & 3.59 \\
\hline Ho-166m & 4603.1 & $1.41 \times 10^{4}$ & $8.00 \times 10^{1}$ \\
\hline $\mathrm{I}-129$ & 805.4 & $2.65 \times 10^{1}$ & 5.41 \\
\hline $\mathrm{K}-40$ & 1340.9 & $7.41 \times 10^{1}$ & 1.03 \\
\hline La-137 & $15188: 6$ & $5.59 \times 10^{4}$ & $1.76 \times 10^{1}$ \\
\hline La-138 & $15785: 3$ & $3.43 \times 10^{-3}$ & $1.48 \times 10^{-5}$ \\
\hline Mn-53 & 2943.4 & $3.97 \times 10^{4}$ & 2.87 \\
\hline Mo-93 & 1103.3 & $9.36 \times 10^{5}$ & $8.88 \times 10^{2}$ \\
\hline $\mathrm{Nb}-94$ & 5951.2 & $3.99 \times 10^{5}$ & $1.49 \times 10^{3}$ \\
\hline $\mathrm{Ni}-59$ & 10283.4 & $3.10 \times 10^{6}$ & $4.52 \times 10^{2}$ \\
\hline $\mathrm{Pb}-202$ & 7884.8 & $3.45 \times 10^{6}$ & $9.81 \times 10^{4}$ \\
\hline $\mathrm{Pb}-205$ & 10534.5 & $4.74 \times 10^{4}$ & $5.20 \times 10^{1}$ \\
\hline Pt-193 & 965.6 & $2.33 \times 10^{2}$ & $1.87 \times 10^{-2}$ \\
\hline Se-79 & 6097 & $3.82 \times 10^{4}$ & $2.32 \times 10^{2}$ \\
\hline Si-32 & 1404.2 & $3.39 \times 10^{3}$ & 4.20 \\
\hline Sm-146 & 10622.9 & $3.19 \times 10^{2}$ & $4.65 \times 10^{1}$ \\
\hline Sm-151 & 2769.6 & $1.23 \times 10^{-4}$ & $3.06 \times 10^{-8}$ \\
\hline Sr-90 & 765.3 & $6.69 \times 10^{-2}$ & $6.35 \times 10^{-3}$ \\
\hline Tb-157 & 2907.3 & $1.53 \times 10^{1}$ & $1.11 \times 10^{-3}$ \\
\hline Tb-158 & 4444.8 & $2.92 \times 10^{6}$ & $8.52 \times 10^{3}$ \\
\hline Tc-97 & 798.8 & $7.94 \times 10^{2}$ & $8.69 \times 10^{-2}$ \\
\hline Tc-98 & 798.8 & $2.54 \times 10^{2}$ & $8.90 \times 10^{-1}$ \\
\hline Tc-99 & 669 & $4.91 \times 10^{4}$ & $4.66 \times 10^{1}$ \\
\hline Ti-44 & 4030.8 & $4.61 \times 10^{-20}$ & $6.40 \times 10^{-22}$ \\
\hline $\mathrm{Zr}-93$ & 10417.5 & $2.18 \times 10^{3}$ & 2.55 \\
\hline
\end{tabular}


Table 4. Radionuclide requiring detailed analysis

\begin{tabular}{|l|r|}
\hline \multicolumn{1}{|c|}{ Radionuclide } & \multicolumn{1}{c|}{$\begin{array}{r}\text { Peak Dose } \\
\text { (mrem/yr) }\end{array}$} \\
\hline Al-26 & $2.73 \times 10^{2}$ \\
\hline Be-10 & 6.82 \\
\hline C-14 & $1.26 \times 10^{4}$ \\
\hline Ca-41 & $5.99 \times 10^{2}$ \\
\hline Cl-36 & $5.90 \times 10^{2}$ \\
\hline Fe-60 & $5.73 \times 10^{1}$ \\
\hline Hf-182 & 3.59 \\
\hline Ho-166m & $8.00 \times 10^{1}$ \\
\hline I-129 & 5.41 \\
\hline K-40 & 1.03 \\
\hline La-137 & $1.76 \times 10^{1}$ \\
\hline Mn-53 & 2.87 \\
\hline Mo-93 & $8.88 \times 10^{2}$ \\
\hline Nb-94 & $1.49 \times 10^{3}$ \\
\hline Ni-59 & $4.52 \times 10^{2}$ \\
\hline Pb-202 & $9.81 \times 10^{4}$ \\
\hline Pb-205 & $5.20 \times 10^{1}$ \\
\hline Se-79 & $2.32 \times 10^{2}$ \\
\hline Si-32 & 4.20 \\
\hline Sm-146 & $4.65 \times 10^{1}$ \\
\hline Tb-158 & $8.52 \times 10^{3}$ \\
\hline Tc-99 & $4.66 \times 10^{1}$ \\
\hline Zr-93 & 2.55 \\
\hline & \\
\hline &
\end{tabular}


References

1. Cook, J. R. and Wilhite, E. L., Radionuclide Screening and Preliminary Scoping Study for EAV Disposal of APT and TEF Wastes, WSRC-RP-98-00084, Westinghouse Savannah River Company, Aiken, SC, 1998.

2. NCRP, Screening Models for Releases of Radionuclides to Atmosphere, Surface Water and Ground, NCRP Report No. 123, National Council on Radiation Protection and Measurements, Bethesda, MD, 1996.

3. Merrell, G. B., Rogers, V. C. and Chau, T. K., The PATHRAE-RAD Performance Assessment Code for the Land Disposal of Radioactive Wastes, RAE-9500/2-1, Rogers and Associates Engineering Corporation, Salt Lake City, UT, 1995.

4. USDOE, Waste Management Activities for Groundwater Protection, Savannah River Plant, Aiken, South Carolina DOE/EIS-0120, U. S. Department of Energy, Washington, SC, 1987.

5. MMES, Radiological Performance Assessment for the E-Area Vaults Disposal Facility, WSRC-RP-94-218 Rev. 0, Martin Marietta Energy Systems, Inc., EG\&G Idaho, Inc and Westinghouse Savannah River Company, Aiken SC, 1994.

6. USDOE, Internal Dose Conversion Factors for Calculation of Dose to the Public, DOE/EH0071, U.S. Department of Energy, Washington, DC, 1988.

7. USEPA, PRESTO-EPA-POP: A Low-Level Radioactive Waste Environmental Transport and Risk Assessment Code - Volume 1, Methodology Manual, EPA 520/1-87-024-1, U.S. Environmental Protection Agency, Washington, DC, 1987.

8. Looney, B. B., King, C. M., and Stephenson, D. E., Environmental Information Document: Quality Assurance Program for Environmental Assessment of Savannah River Plant Waste Sites, E. I. du Pont de Nemours \& Co., Savannah River Laboratory, Aiken, SC, March 1987. 
9. Chandler, G. T., Corrosion Evaluation of Accelerator Production of Tritium and Tritium Extraction Facility Low-Level Waste Forms and Disposal Boxes in the E-Area Vaults, SRTCMTS-98-2028, Savannah River Technology Center, Aiken, SC, August 7, 1998.

10. Sheppard, Marsha I. and Thibault, D. H., Default Soil Solid/Liquid Partition Coefficients, $\mathbf{K}_{d} \mathbf{s}$, for Four Major Soil Types: A Compendium, Health Physics, Vol. 59, No. 4, 1990.

11. Baes, C. F., III and Sharp, R. D., A Proposal for Estimation of Soil Leaching or Leaching Constant for use in Assessment Models, Journal of Environmental Quality, Vol. 12, No. 17, 1983.

12. Kennedy, W. E. and Strenge, D. L., Residual Radioactive Contamination from Decommissioning, NUREG/CR-5512, U. S. Nuclear Regulatory Commission, Washington, DC, 1992.

13. McIntyre, P. F., Sorption Properties of Carbon-14 on Savannah River Plant Soil, DPST-88900, Savannah River Laboratory, Aiken, SC, 1988.

14. Hoeffner, Steven L., Radionuclide Sorption on Savannah River Plant Burial Ground Soil - A Summary and Interpretation of Laboratory Data, DP-1702, Savannah River Laboratory, Aiken, SC, 1985. 
B. T. Butcher

\section{ATTACHMEN'T I}

OUTPUT FROM PATHRAE COMPUTER PROGRAM 


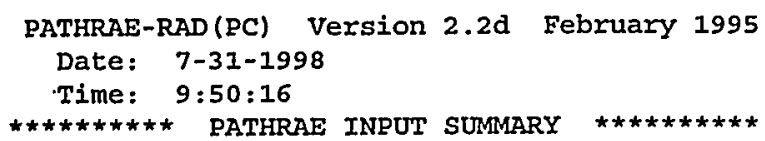

THERE ARE 80 ISOTOPES IN THE DOSE FACTOR LIBRARY NUMBER OF TIMES FOR CALCULATION IS 2

yEARS TO BE CALCULATED ARE ...

.0010000 .00

THERE ARE 59 ISOTORES IN THE INVENTORY FILE

THE VALUE OF IFLAG IS 0

NUMBER OF PATHWAYS IS 1

PATHWAY

TYPE OF USAGE FOR UPTAKE FACTORS

2 GROUNDWATER TO WELL

TIME OF OPERATION OF WASTE FACILITY IN YEARS

LENGTH OF REPOSITORY (M)

WIDTH OF REPOSITORY (M)

RIVER FLOW RATE $(M \star \star 3 / Y R)$

STREAM FLOW RATE $(M * * 3 / Y R)$

DISTANCE TO RIVER (M)

OPERATIONAI SPILLAGE FRACTION

DENSITY OF AQUIFER (KG/M**3)

LONGITUDINAL DISPERSIVITY (M)

LATERAL DISPERSION COEFFICIENT -- Y AXIS (M**2/YR)

NUMBER OF MESH POINTS FOR DISPERSION CALCULATION

FLAG EOR GAMMA PATHWAY OPTIONS

FLAG FOR GAMMA BUILDUP CALCULATION

FLAG FOR ATMOSPHERIC PATHWAY

COVER THICKNESS OVER WASTE (M)

THICKNESS OF WASTE IN PITS (M)

TOTAI WASTE VOLUME $(M * * 3)$

DISTANCE TO WELL -- X COORDINATE (M)

DISTANCE TO WELL - - Y COORDINATE (M)

DENSITY OF WASTE $(\mathrm{KG} / \mathrm{M} * \star 3)$

ERACTION OF FOOD CONSUMED THAT IS GROWN ON SITE FRACTION OF YEAR SPENT IN DIRECT RADIATION FIELD

DEPTH OF PLANT ROOT ZONE (M)

AREAL DENSITY OF PLANTS (KG/M**2)

AVERAGE DUST LOADING IN AIR (KG/M**3)

ANNUAL ADULT BREATHING RATE $(M * * 3 / Y R)$

FRACTION OF YEAR EXPOSED TO DUST

CANISTER LIFETIME (YEARS)

INVENTORY SCALING FACTOR

HEIGHT OF ROOMS IN RECLAIMER HOUSE (CM)

AIR CHANGE RATE IN RECLAIMER HOUSE (CHANGES/SEC) O RADON EMANATING POWER OF THE WASTE

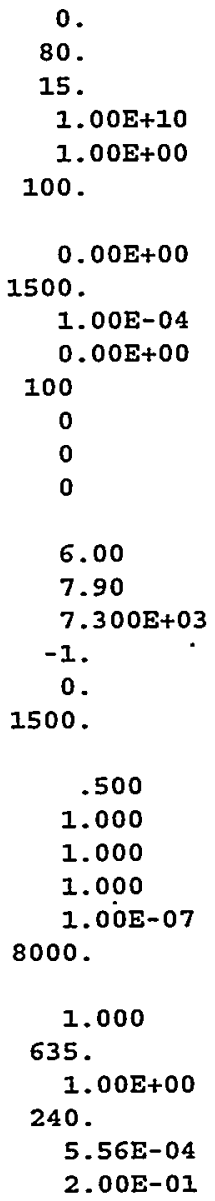


DIFFUSION COEFF. OF RADON IN WASTE (CM**2/SEC)

DIFFUSION COEFF. OF RN IN CONCRETE (CM**2/SEC)

THICKNESS OF CONCRETE SLAB FLOOR (CM)

DIFFUSION COEFF. OF RADON IN COVER (CM**2/SEC)

ATMOSPHERIC STABILITY CLASS

AVERAGE WIND SPEED (M/S)

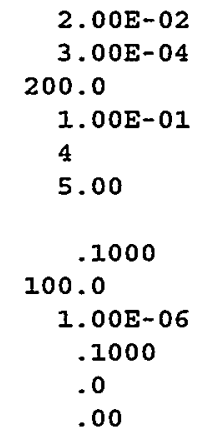

.00

FRACTION OF TIME WIND BLOWS TOWARD RECEPTOR

RECEPTOR DISTANCE FOR ATMOSPHERIC PATHWAY (M)

DUST RESUSPENSION RATE FOR OFFSITE TRANSPORT $(M * * 3 / S)$

DEPOSITION VELOCITY (M/S)

STACK HEIGHT (M)

STACK INSIDE DIAMETER (M)

STACK GAS VELOCITY (M/S)

HEAT EMISSION RATE FROM BURNING (CAL/S)

DECAY CHAIN FLAGS

FLAG FOR INPUT SUMMARY PRINTOUT

FLAG FOR DIRECTION OF TRENCH FILLING

FLAG FOR GROUNDWATER PATHWAY OPTIONS
.0
$0.00 \mathrm{E}+00$
$\begin{array}{lllllll}0 & 0 & 0 & 0 & 0 & 0 & 0\end{array}$
1
1
1

AMOUNT OF WATER PERCOLATING THROUGH WASTE ANNUALLY (M)

DEGREE OF SOIL SATURATION

RESIDUAL SOIL SATURATION

PERMEABILITY OF VERTICAI ZONE (M/YR)

SOIL NUMBER

POROSITY OF AQUIFER

4.00E-01

.500

.100

10.00

.250

.30

POROSITY OF UNSATURATED ZONE

DISTANCE FROM AQUIFER TO WASTE (M)

.30

AVERAGE VERTICAL GROUNDWATER VELOCITY (M/YR)

HORIZONTAL VELOCITY OF AQUIFER (M/YR)

8.0

$1.00 \mathrm{E}+01$

LENGTH OF PERFORATED WELL CASING (M)

SURFACE EROSION RATE (M/YR)

10.0

10.000

LEACH RATE SCALING FACTOR

$2.000 \mathrm{E}-04$

$1.000 \mathrm{E}+00$

ANNUAL RUNOFF OF PRECIPITATION (M)

$5.00 \mathrm{E}-01$ 


\begin{tabular}{|c|c|c|c|c|}
\hline NUCLIDE & $\begin{array}{l}\text { DOSE FACTORS } \\
\text { (MREM/PCI) }\end{array}$ & $\begin{array}{l}\text { DOSE FACTORS } \\
\text { (MREM/PCI) }\end{array}$ & $\begin{array}{l}\text { DOSE FACTORS } \\
\text { (MREM-M2/PCI-YR) }\end{array}$ & $\begin{array}{c}\text { HALF } \\
\text { LIFE (YR) }\end{array}$ \\
\hline $\mathrm{Ag}-108 \mathrm{~m}$ & $7.500 \mathrm{E}-06$ & $2.000 \mathrm{E}-04$ & $1.700 E-04$ & $1.270 \mathrm{E}+02$ \\
\hline$A]-26$ & $7.900 \mathrm{E}-06$ & $5.900 \mathrm{E}-06$ & $2.500 \mathrm{E}-04$ & $7.200 \mathrm{E}+05$ \\
\hline Ar-39 & $0.000 \mathrm{E}+00$ & $0.000 \mathrm{E}+00$ & $0.000 \mathrm{E}+00$ & $2.690 \mathrm{E}+02$ \\
\hline$A x-42$ & $0.000 \mathrm{E}+00$ & $0.000 \mathrm{E}+00$ & $0.000 \mathrm{E}+00$ & $3.300 \mathrm{E}+01$ \\
\hline $\mathrm{Ba}-133$ & $3.200 \mathrm{E}-06$ & $6.900 \mathrm{E}-06$ & $6.200 \mathrm{E}-05$ & $1.050 \mathrm{E}+01$ \\
\hline $\mathrm{Be}-10$ & $4.200 \mathrm{E}-06$ & $2.700 \mathrm{E}-07$ & $0.000 \mathrm{E}+00$ & $1.600 \mathrm{E}+06$ \\
\hline$B i-207$ & $4.900 E-06$ & $1.400 \mathrm{E}-05$ & $1.500 E-04$ & $3.340 \mathrm{E}+01$ \\
\hline$C-14$ & $2.100 \mathrm{E}-06$ & $2.100 \mathrm{E}-06$ & $0.000 E \div 00$ & $5.730 \mathrm{E}+03$ \\
\hline $\mathrm{Ca}-41$ & 1.200E-06 & $1.300 \mathrm{E}-06$ & $2.1005-09$ & $1.030 \mathrm{E}+05$ \\
\hline$C d-113 m$ & 1. $500 \mathrm{E}-04$ & $1.400 \mathrm{E}-03$ & $0.000 E+00$ & $1.370 \mathrm{E}+01$ \\
\hline $\mathrm{Cl}-36$ & $3.000 \mathrm{E}-06$ & $2.000 \mathrm{E}-05$ & $4.700 \mathrm{E}-12$ & $3.010 \mathrm{E}+05$ \\
\hline Co-60 & $2.600 \mathrm{E}-05$ & $1.500 \mathrm{E}-04$ & $2.300 E-04$ & $5.270 \mathrm{E}+00$ \\
\hline $\mathrm{Cs}-135$ & $7.100 \mathrm{E}-06$ & $4.500 \mathrm{E}-06$ & $0.000 \mathrm{E}+00$ & $2.300 \mathrm{E}+06$ \\
\hline $\mathrm{Cs}-1.37$ & $5.000 \mathrm{E}-05$ & $3.200 \mathrm{E}-05$ & $6.100 \mathrm{E}-05$ & $3.020 \mathrm{E}+01$ \\
\hline Eu-150 & $1.500 \mathrm{E}-06$ & $6.100 \mathrm{E}-07$ & $0.000 E+00$ & $5.000 \mathrm{E}+00$ \\
\hline $\mathrm{Eu}-152$ & $6.000 \mathrm{E}-06$ & $2.200 \mathrm{E}-04$ & $1.100 \mathrm{E}-04$ & $1.360 \mathrm{E}+01$ \\
\hline $\mathrm{Eu}-154$ & $9.100 \mathrm{E}-06$ & $2.600 \mathrm{E}-04$ & 1. $200 E-04$ & $8.800 \mathrm{E}+00$ \\
\hline $\mathrm{Fe}-60$ & $1.500 \mathrm{E}-04$ & $7.600 \mathrm{E}-04$ & $0.000 E+00$ & $3.000 \mathrm{E}+05$ \\
\hline Gd-148 & $2.100 \mathrm{E}-04$ & $3.300 \mathrm{E}-01$ & $0.000 \mathrm{E}+00$ & $8.400 \mathrm{E}+01$ \\
\hline $\mathrm{Ge}-68$ & $1.100 \mathrm{E}-06$ & $4.900 \mathrm{E}-05$ & 1. $200 \mathrm{E}-07$ & $7.900 \mathrm{E}-01$ \\
\hline $\mathrm{H}-3$ & $6.300 \mathrm{E}-08$ & $6.300 \mathrm{E}-08$ & $0.000 \mathrm{E}+00$ & $1.230 \mathrm{E}+01$ \\
\hline Hf- $178 \mathrm{~m}$ & $2.000 \mathrm{E}-05$ & $2.000 \mathrm{E}-03$ & $0.000 \mathrm{E}+00$ & $1.360 \mathrm{E}-07$ \\
\hline $\mathrm{HE}-182$ & $1.400 \mathrm{E}-05$ & $2.800 \mathrm{E}-03$ & $0.000 \mathrm{E}+00$ & $9.000 \mathrm{E}+06$ \\
\hline Ho- $-166 \mathrm{~m}$ & $7.800 \mathrm{E}-06$ & $7.200 \mathrm{E}-04$ & 1. $600 \mathrm{E}-04$ & $1.200 \mathrm{E}+03$ \\
\hline$I-129$ & $2.800 \mathrm{E}-04$ & $1.800 \mathrm{E}-04$ & $2.200 \mathrm{E}-06$ & $1.570 \mathrm{E}+07$ \\
\hline$K-40$ & 1.900 E-05 & $1.200 \mathrm{E}-05$ & $1.400 \mathrm{E}-05$ & $1.280 \mathrm{E}+09$ \\
\hline $\mathrm{Kr}-85$ & $0.000 E+00$ & $0.000 E+00$ & $2.300 \mathrm{E}-07$ & $1.070 \mathrm{E}+01$ \\
\hline La-137 & $4.300 \mathrm{E}-07$ & $7.300 \mathrm{E}-05$ & $0.000 \mathrm{E}+00$ & $6.000 \mathrm{E}+04$ \\
\hline La-138 & $5.900 \mathrm{E}-06$ & $1.400 E-03$ & $0.000 \mathrm{E}+00$ & $1.100 \mathrm{E}+11$ \\
\hline$M n-53$ & $9.900 E-08$ & $4.300 \mathrm{E}-07$ & $1.600 \mathrm{E}-08$ & $3.700 \mathrm{E}+06$ \\
\hline Mo-93 & $1.300 \mathrm{E}-06$ & $2.800 \mathrm{E}-05$ & $5.800 \mathrm{E}-07$ & $3.500 \mathrm{E}+03$ \\
\hline $\mathrm{Na}-22$ & $1.200 \mathrm{E}-05$ & $8.000 E-06$ & $2.100 \mathrm{E}-04$ & $2.600 \mathrm{E}+00$ \\
\hline $\mathrm{Nb}-93 \mathrm{~m}$ & $5.300 E-07$ & $2.800 \mathrm{E}-05$ & $1.000 \mathrm{E}-07$ & $1.460 \mathrm{E}+01$ \\
\hline $\mathrm{Nb}-94$ & $5.100 \mathrm{E}-06$ & $3.300 \mathrm{E}-04$ & $1.600 \mathrm{E}-04$ & $2.030 \mathrm{E}+04$ \\
\hline$N i-59$ & $2.000 E-07$ & $2.700 \mathrm{E}-06$ & $4.200 E-08$ & $7.500 \mathrm{E}+04$ \\
\hline$N i-63$ & $5.400 \mathrm{E}-07$ & $6.300 \mathrm{E}-06$ & $0.000 \mathrm{E}+00$ & $1.000 E+02$ \\
\hline $\mathrm{pb}-202$ & $3.900 E-05$ & $9.900 \mathrm{E}-05$ & $0.000 \mathrm{E}+00$ & $3.000 \mathrm{E}+05$ \\
\hline $\mathrm{Pb}-205$ & $1.500 \mathrm{E}-06$ & $3.700 \mathrm{E}-06$ & $7.600 \mathrm{E}-08$ & $1.510 \mathrm{E}+07$ \\
\hline$P m-145$ & $4.600 E-07$ & $2.700 \mathrm{E}-05$ & $2.900 \mathrm{E}-06$ & $1.770 \mathrm{E}+01$ \\
\hline pt-193 & $1.100 \mathrm{E}-07$ & $2.100 \mathrm{E}-07$ & $5.600 \mathrm{E}-08$ & $5.000 \mathrm{E}+01$ \\
\hline Rh-I0I & $2.300 \mathrm{E}-06$ & $3.200 \mathrm{E}-05$ & $0.000 E+00$ & $5.000 E+00$ \\
\hline $\mathrm{Rh}-102 \mathrm{~m}$ & $3.500 E-06$ & $4.200 E-05$ & $0.000 \mathrm{E}+00$ & $5.750 E-01$ \\
\hline$R h-102$ & $8.500 \mathrm{E}-06$ & $8.700 \mathrm{E}-05$ & $0.000 \mathrm{E}+00$ & $2.900 \mathrm{E}+00$ \\
\hline $\mathrm{Se}-79$ & $8.300 \mathrm{E}-06$ & $8.900 \mathrm{E}-06$ & $0.000 \mathrm{E}+00$ & $6.500 \mathrm{E}+04$ \\
\hline$S i-32$ & 1.7000E-06 & $1.000 \mathrm{E}-03$ & $0.000 \mathrm{E}+00$ & $3.300 \mathrm{E}+02$ \\
\hline$S m-146$ & $2.000 \mathrm{E}-04$ & $7.800 \mathrm{E}-02$ & $0.000 \mathrm{E}+00$ & $7.000 \mathrm{E}+07$ \\
\hline$S m-151$ & $3.400 \mathrm{E}-07$ & $2.900 \mathrm{E}-05$ & $5.200 \mathrm{E}-10$ & $9.000 \mathrm{E}+01$ \\
\hline$s n-121 m$ & $1.300 \mathrm{E}-06$ & $8.900 \mathrm{E}-06$ & $0.000 E+00$ & $7.600 E+01$ \\
\hline Sr-90 & $1.300 \mathrm{E}-04$ & $1.300 \mathrm{E}-03$ & $0.000 E+00$ & $2.860 \mathrm{E}+01$ \\
\hline$T b-157$ & $1.000 \mathrm{E}-07$ & $9.000 \mathrm{E}-06$ & $4.700 \mathrm{E}-07$ & $1.500 \mathrm{E}+02$ \\
\hline$T b-158$ & $4.000 \mathrm{E}-06$ & $2.500 \mathrm{E}-04$ & $0.000 E+00$ & $1.200 E+03$ \\
\hline TC-97 & 1. 500E-07 & $8.900 \mathrm{E}-07$ & $6.400 \mathrm{E}-07$ & $2.600 \mathrm{E}+06$ \\
\hline TC-98 & $4.800 \mathrm{E}-06$ & $1.700 \mathrm{E}-05$ & $1.400 \mathrm{E}-04$ & $4.200 E+06$ \\
\hline TC-99 & $1.300 \mathrm{E}-06$ & $7.500 \mathrm{E}-06$ & $6.300 \mathrm{E}-11$ & $2.130 \mathrm{E}+05$ \\
\hline$T i-44$ & $1.900 \mathrm{E}-05$ & $8.900 E-04$ & $1.700 \mathrm{E}-05$ & $4.730 E+01$ \\
\hline$T 1-204$ & $3.200 \mathrm{E}-06$ & $2.300 E-06$ & $1.300 \mathrm{E}-07$ & $3.780 \mathrm{E}+00$ \\
\hline$v-49$ & $5.400 \mathrm{E}-08$ & $2.800 \mathrm{E}-07$ & $7.500 \mathrm{E}-09$ & $9.000 \mathrm{E}-01$ \\
\hline$W-181$ & $3.100 \mathrm{E}-07$ & $1.500 \mathrm{E}-07$ & $4.700 \mathrm{E}-06$ & $3.300 \mathrm{E}-01$ \\
\hline$z x-93$ & $1.600 \mathrm{E}-06$ & $3.200 \mathrm{E}-04$ & $0.000 E+00$ & $1.530 \mathrm{E}+06$ \\
\hline
\end{tabular}




\begin{tabular}{|c|c|c|c|}
\hline NUCLIDE & $\begin{array}{l}\text { VOLATILITY } \\
\text { FRACTION }\end{array}$ & $\begin{array}{c}\text { GAMMA } \\
\text { ENERGY } \\
\text { (MEV) }\end{array}$ & $\begin{array}{c}\text { GAMMA } \\
\text { ATTENUATION } \\
(I / M)\end{array}$ \\
\hline $\mathrm{Ag}-108 \mathrm{~m}$ & $0.000 \mathrm{E}+00$ & $4.710 \mathrm{E}-01$ & $1.440 E+01$ \\
\hline Al-26 & $0.000 \mathrm{E}+00$ & $9.940 \mathrm{E}-01$ & $1.010 \mathrm{E}+01$ \\
\hline Ar-39 & $0.000 \mathrm{E}+00$ & $0.000 \mathrm{E}+00$ & $0.000 E+00$ \\
\hline Ar -42 & $0.000 \mathrm{E}+00$ & $0.000 \mathrm{E}+00$ & $0.000 \mathrm{E}+00$ \\
\hline $\mathrm{Ba}-133$ & $0.000 \mathrm{E}+00$ & $1.540 \mathrm{E}-01$ & $2.220 E+01$ \\
\hline $\mathrm{Be}-10$ & $0.000 \mathrm{E}+00$ & $0.000 \mathrm{E}+00$ & $0.000 E+00$ \\
\hline $\mathrm{Bi}-207$ & $0.000 \mathrm{E}+00$ & $8.350 E-01$ & 1. $110 E+01$ \\
\hline$C-14$ & $0.000 \mathrm{E}+00$ & $0.000 \mathrm{E}+00$ & $0.000 \mathrm{E}+00$ \\
\hline $\mathrm{Ca}-41$ & $0.000 \mathrm{E}+00$ & $3.000 \mathrm{E}-03$ & $5.000 E+02$ \\
\hline $\mathrm{Cd}-113 \mathrm{~m}$ & $0.000 \mathrm{E}+00$ & $0.000 \mathrm{E}+00$ & $0.000 \mathrm{E}+00$ \\
\hline $\mathrm{Cl}-36$ & $0.000 \mathrm{E}+00$ & $5.110 \mathrm{E}-01$ & I. $390 \mathrm{E}+01$ \\
\hline Co- 60 & $0.000 \mathrm{E}+00$ & $1.253 \mathrm{E}+00$ & $9.200 \mathrm{E}+00$ \\
\hline Cs-135 & $0.000 \mathrm{E}+00$ & $0.000 \mathrm{E}+00$ & $0.000 \mathrm{E}+00$ \\
\hline Cs-137 & $0.000 \mathrm{E}+00$ & $0.000 \mathrm{E}+00$ & $0.000 \mathrm{E}+00$ \\
\hline Eu-150 & $0.000 \mathrm{E}+00$ & $5.030 \mathrm{E}-01$ & $1.390 \mathrm{E}+01$ \\
\hline Eu-152 & $0.000 \mathrm{E}+00$ & $4.970 \mathrm{E}-01$ & $1.400 \mathrm{E}+01$ \\
\hline Eu-154 & $0.000 \mathrm{E}+00$ & $6.570 \mathrm{E}-01$ & $1.250 \mathrm{E}+01$ \\
\hline $\mathrm{Fe}-60$ & $0.000 \mathrm{E}+00$ & $0.000 \mathrm{E}+00$ & $0.000 \mathrm{E}+00$ \\
\hline Gd-148 & $0.000 \mathrm{E}+00$ & $0.000 \mathrm{E}+00$ & $0.000 \mathrm{E}+00$ \\
\hline $\mathrm{Ge}-68$ & $0.000 \mathrm{E}+00$ & $0.000 \mathrm{E}+00$ & $0.000 \mathrm{E}+00$ \\
\hline $\mathrm{H}-3$ & $0.000 \mathrm{E}+00$ & $0.000 \mathrm{E}+00$ & $0.000 \mathrm{E}+00$ \\
\hline $\mathrm{Hf}-178 \mathrm{~m}$ & $0.000 \mathrm{E}+00$ & $0.000 \mathrm{E}+00$ & $0.000 \mathrm{E}+00$ \\
\hline Hf -182 & $0.000 \mathrm{E}+00$ & $2.710 \mathrm{E}-01$ & 1. $770 \mathrm{E}+01$ \\
\hline Ho- $166 \mathrm{~m}$ & $0.000 \mathrm{E}+00$ & $4.600 \mathrm{E}-01$ & $1.450 \mathrm{E}+01$ \\
\hline$I-129$ & $0.000 \mathrm{E}+00$ & $3.100 \mathrm{E}-02$ & $1.310 \mathrm{E}+02$ \\
\hline $\mathrm{K}-40$ & $0.000 \mathrm{E}+00$ & $9.860 \mathrm{E}-01$ & $1.030 \mathrm{E}+01$ \\
\hline$K x-85$ & $0.000 \mathrm{E}+00$ & $5.140 \mathrm{E}-01$ & $1.380 \mathrm{E}+01$ \\
\hline La-137 & $0.000 \mathrm{E}+00$ & $0.000 \mathrm{E}+00$ & $0.000 \mathrm{E}+00$ \\
\hline La-138 & $0.000 \mathrm{E}+00$ & $1.240 \mathrm{E}+00$ & $9.270 E+00$ \\
\hline$M n-53$ & $0.000 \mathrm{E}+00$ & $0.000 \mathrm{E}+00$ & $0.000 \mathrm{E}+00$ \\
\hline Mo-93 & $0.000 \mathrm{E}+00$ & $0.000 \mathrm{E}+00$ & $0.000 \mathrm{E}+00$ \\
\hline $\mathrm{Na}-22$ & $0.000 \mathrm{E}+00$ & $7.840 \mathrm{E}-0 \mathrm{I}$ & $1.140 \mathrm{E}+01$ \\
\hline $\mathrm{Nb}-93 \mathrm{~m}$ & $0.000 \mathrm{E}+00$ & $1.420 \mathrm{E}-02$ & $3.300 \mathrm{E}+03$ \\
\hline $\mathrm{Nb}-94$ & $0.000 \mathrm{E}+00$ & $7.870 \mathrm{E}-01$ & $1.140 \mathrm{E}+01$ \\
\hline $\mathrm{Ni}-59$ & $0.000 \mathrm{E}+00$ & $0.000 E+00$ & $0.000 \mathrm{E}+00$ \\
\hline $\mathrm{Ni}-63$ & $0.000 \mathrm{E}+00$ & $0.000 E+00$ & $0.000 E+00$ \\
\hline $\mathrm{Pb}-202$ & $0.000 \mathrm{E}+00$ & $0.000 E+00$ & $0.000 E+00$ \\
\hline $\mathrm{Pb}-205$ & $0.000 E+00$ & $1.000 \mathrm{E}-02$ & $5.060 \mathrm{E}+03$ \\
\hline $\mathrm{Pm}-145$ & $0.000 \mathrm{E}+00$ & $3.390 \mathrm{E}-02$ & $1.020 \mathrm{E}+02$ \\
\hline Pt -193 & $0.000 E+00$ & $9.000 \mathrm{E}-03$ & $4.360 \mathrm{E}+03$ \\
\hline $\mathrm{Rh}-101$ & $0.000 \mathrm{E}+00$ & $1.700 \mathrm{E}-01$ & $2.080 \mathrm{E}+01$ \\
\hline $\mathrm{Rh}-102 \mathrm{~m}$ & $0.000 \mathrm{E}+00$ & $5.200 \mathrm{E}-01$ & $1.370 \mathrm{E}+01$ \\
\hline Rh-102 & $0.000 E+00$ & $6.890 \mathrm{E}-01$ & 1. $220 \mathrm{E}+01$ \\
\hline Se-79 & $0.000 E+00$ & $0.000 \mathrm{E}+00$ & $0.000 \mathrm{E}+00$ \\
\hline Si-32 & $0.000 E+00$ & $0.000 \mathrm{E}+00$ & $0.000 \mathrm{E}+00$ \\
\hline $\mathrm{Sm}-146$ & $0.000 \mathrm{E}+00$ & $0.000 \mathrm{E}+00$ & $0.000 \mathrm{E}+00$ \\
\hline$S m-151$ & $0.000 \mathrm{E}+00$ & $2.200 \mathrm{E}-02$ & $3.460 \mathrm{E}+02$ \\
\hline $\mathrm{Sn}-121 \mathrm{~m}$ & $0.000 \mathrm{E}+00$ & $0.000 \mathrm{E}+00$ & $0.000 \mathrm{E}+00$ \\
\hline Sr-90 & $0.000 \mathrm{E}+00$ & $0.000 \mathrm{E}+00$ & $0.000 \mathrm{E}+00$ \\
\hline $\mathrm{Tb}-157$ & $0.000 \mathrm{E}+00$ & $2.120 \mathrm{E}-02$ & $3.380 \mathrm{E}+02$ \\
\hline $\mathrm{Tb}-158$ & $0.000 \mathrm{E}+00$ & $7.690 \mathrm{E}-01$ & $1.160 \mathrm{E}+01$ \\
\hline TC-97 & $0.000 \mathrm{E}+00$ & $1.660 \mathrm{E}-02$ & $7.260 E+02$ \\
\hline TC-98 & $0.000 \mathrm{E}+00$ & $6.960 \mathrm{E}-01$ & $1.210 \mathrm{E}+01$ \\
\hline Tc-99 & $0.000 E+00$ & $8.900 E-02$ & $2.920 E+01$ \\
\hline $\mathrm{Ti}-44$ & $0.000 E+00$ & $6.700 \mathrm{E}-02$ & $3.340 E+01$ \\
\hline Tl-204 & $0.000 \mathrm{E}+00$ & $5.100 E-02$ & $4.420 E+01$ \\
\hline$V-49$ & $0.000 \mathrm{E}+00$ & $4.490 \mathrm{E}-03$ & $5.420 E+01$ \\
\hline$W-181$ & $0.000 E+00$ & $4.560 \mathrm{E}-02$ & $5.420 E+01$ \\
\hline$z x-93$ & $0.000 \mathrm{E}+00$ & $0.000 E+00$ & $0.000 \mathrm{E}+00$ \\
\hline
\end{tabular}




\begin{tabular}{|c|c|c|c|c|c|}
\hline NUCLIDE & & $\begin{array}{l}\text { INPUT IEACH } \\
\text { RATE (1/YR) }\end{array}$ & $\begin{array}{l}\text { FINAL LEACH } \\
\text { RATE (I/YR) }\end{array}$ & $\begin{array}{l}\text { SOLUBILITY } \\
\text { (MOLE/L) }\end{array}$ & $\begin{array}{c}\text { INPUT } \\
\text { INVENTORY } \\
\text { (CI) }\end{array}$ \\
\hline $\mathrm{Ag}-108 \mathrm{~m}$ & & $2.540 \mathrm{E}-04$ & $2.540 \mathrm{E}-04$ & $0.000 \mathrm{E}+00$ & $3.500 \mathrm{E}+01$ \\
\hline Al-26 & & $2.540 \mathrm{E}-04$ & $2.540 E-04$ & $0.000 \mathrm{E}+00$ & $1.300 \mathrm{E}+03$ \\
\hline$A x-39$ & & $2.540 \mathrm{E}-04$ & $2.540 E-04$ & $0.000 E+00$ & $1.500 \mathrm{E}+05$ \\
\hline Ar -42 & & $2.540 \mathrm{E}-04$ & $2.540 \mathrm{E}-04$ & $0.000 \mathrm{E}+00$ & $1.500 \mathrm{E}+04$ \\
\hline $\mathrm{Ba}-133$ & & $2.540 \mathrm{E}-04$ & $2.540 \mathrm{E}-04$ & $0.000 \mathrm{E}+00$ & $3.900 E+06$ \\
\hline $\mathrm{Be}-10$ & & $2.540 \mathrm{E}-04$ & $2.540 \mathrm{E}-04$ & $0.000 \mathrm{E}+00$ & $1.000 E+01$ \\
\hline $\mathrm{Bi}-207$ & * & $2.540 \mathrm{E}-04$ & $2.540 \mathrm{E}-04$ & $0.000 \mathrm{E}+00$ & $1.000 \mathrm{E}+06$ \\
\hline$C-14$ & & $2.540 \mathrm{E}-04$ & $2.540 \mathrm{E}-04$ & $0.000 \mathrm{E}+00$ & $1.700 \mathrm{E} \div 04$ \\
\hline $\mathrm{Ca}-41$ & & $2.540 E-04$ & $2.540 \mathrm{E}-04$ & $0.000 \mathrm{E}+00$ & $1.300 \mathrm{E}+03$ \\
\hline $\mathrm{cd}-1.13 \mathrm{~m}$ & & $2.540 \mathrm{E}-04$ & $2.540 \mathrm{E}-04$ & $0.000 \mathrm{E}+00$ & $2.300 E+02$ \\
\hline $\mathrm{Cl-36}$ & & $2.540 \mathrm{E}-04$ & $2.540 \mathrm{E}-04$ & $0.000 \mathrm{E}+00$ & $5.100 \mathrm{E}+02$ \\
\hline Co- 60 & & $2.540 \mathrm{E}-04$ & $2.540 \mathrm{E}-04$ & $0.000 E+00$ & $3.600 E+07$ \\
\hline $\mathrm{Cs}-1.35$ & & $2.540 \mathrm{E}-04$ & $2.540 \mathrm{E}-04$ & $0.000 E+00$ & $2.800 \mathrm{E}-01$ \\
\hline $\operatorname{Cs}-137$ & & $2.540 \mathrm{E}-04$ & $2.540 E-04$ & $0.000 \mathrm{E}+00$ & $1.200 \mathrm{E}+0 \mathrm{I}$ \\
\hline $\mathrm{Eu}-150$ & & $2.540 \mathrm{E}-04$ & $2.540 \mathrm{E}-04$ & $0.000 E+00$ & $2.000 \mathrm{E}+05$ \\
\hline$E u-152$ & & $2.540 \mathrm{E}-04$ & $2.540 \mathrm{E}-04$ & $0.000 E+00$ & $1.300 \mathrm{E}+06$ \\
\hline Eu-154 & & $2.540 \mathrm{E}-04$ & $2.540 \mathrm{E}-04$ & $0.000 \mathrm{E}+00$ & $1.300 \mathrm{E}+06$ \\
\hline $\mathrm{Fe}-60$ & & $2.540 \mathrm{E}-04$ & $2.54 \mathrm{OE}-04$ & $0.000 E+00$ & $2.100 E+00$ \\
\hline Gd-148 & & $2.540 \mathrm{E}-04$ & $2.540 \mathrm{E}-04$ & $0.000 E+00$ & $1.400 E+06$ \\
\hline $\mathrm{Ge}-68$ & & $2.540 \mathrm{E}-04$ & $2.540 E-04$ & $0.000 E+00$ & $5.600 \mathrm{E}+04$ \\
\hline $\mathrm{H}-3$ & & $2.540 \mathrm{E}-04$ & $2.540 \mathrm{E}-04$ & $0.000 E+00$ & $2.300 E+09$ \\
\hline $\mathrm{Hf}-178 \mathrm{~m}$ & & $2.540 \mathrm{E}-04$ & $2.540 \mathrm{E}-04$ & $0.000 \mathrm{E}+00$ & $4.300 \mathrm{E}+02$ \\
\hline Hf -1.82 & & $2.540 \mathrm{E}-04$ & $2.540 E-04$ & $0.000 \mathrm{E} \div 00$ & $2.800 \mathrm{E}+00$ \\
\hline Ho- $-166 \mathrm{~m}$ & & $2.540 \mathrm{E}-04$ & $2.540 E-04$ & $0.000 \mathrm{E}+00$ & $1.900 \mathrm{E}+03$ \\
\hline I-129 & & $2.540 \mathrm{E}-04$ & $2.540 \mathrm{E}-04$ & $0.000 E+00$ & $5.000 \mathrm{E}-02$ \\
\hline $\mathrm{K}-40$ & & $2.540 E-04$ & $2.540 E-04$ & $0.000 E+00$ & $1.400 \mathrm{E}-01$ \\
\hline $\mathrm{Kr}-85$ & & $2.540 \mathrm{E}-04$ & $2.540 \mathrm{E}-04$ & $0.000 \mathrm{E}+00$ & $1.200 \mathrm{E}+05$ \\
\hline La-137 & & $2.540 \mathrm{E}-04$ & $2.540 E-04$ & $0.000 E+00$ & $1.400 \mathrm{E}+03$ \\
\hline La-1.38 & & $2.540 E-04$ & $2.540 \mathrm{E}-04$ & $0.000 \mathrm{E}+00$ & $7.200 \mathrm{E}-05$ \\
\hline $\mathrm{Mn}-53$ & & $2.540 \mathrm{E}-04$ & $2.540 \mathrm{E}-04$ & $0.000 \mathrm{E}+00$ & $7.500 \mathrm{E}+01$ \\
\hline Mo-93 & & $2.540 \mathrm{E}-04$ & $2.540 \mathrm{E}-04$ & $0.000 E+00$ & $2.200 \mathrm{E}+03$ \\
\hline $\mathrm{Na}-22$ & & $2.540 E-04$ & $2.540 \mathrm{E}-04$ & $0.000 E+00$ & $2.200 E+07$ \\
\hline $\mathrm{Nb}-93 \mathrm{~m}$ & & $2.540 \mathrm{E}-04$ & $2.540 E-04$ & $0.000 \mathrm{E}+00$ & $6.800 E+05$ \\
\hline $\mathrm{Nb}-94$ & & $2.540 \mathrm{E}-04$ & $2.540 E-04$ & $0.000 E+00$ & $1.400 \mathrm{E}+03$ \\
\hline $\mathrm{Ni}-59$ & & $2.540 \mathrm{E}-04$ & $2.540 \mathrm{E}-04$ & $1.000 \mathrm{E}-02$ & $2.400 E+04$ \\
\hline$N i-63$ & & $2.540 \mathrm{E}-04$ & $2.540 \mathrm{E}-04$ & $1.000 \mathrm{E}-02$ & $2.800 E+06$ \\
\hline $\mathrm{Pb}-202$ & & $2.540 \mathrm{E}-04$ & $2.540 \mathrm{E}-04$ & $0.000 E+00$ & $1.700 \mathrm{E}+04$ \\
\hline $\mathrm{Pb}-205$ & & $2.540 \mathrm{E}-04$ & $2.540 \mathrm{E}-04$ & $0.000 \mathrm{E}+00$ & $2.300 E+02$ \\
\hline $\mathrm{Pm}-145$ & & $2.540 \mathrm{E}-04$ & $2.540 \mathrm{E}-04$ & $0.000 \mathrm{E}+00$ & $5.200 \mathrm{E}+06$ \\
\hline Pt -193 & $\cdot$ & $2.540 \mathrm{E}-04$ & $2.540 \mathrm{E}-04$ & $0.000 \mathrm{E}+00$ & $4.100 E+06$ \\
\hline $\mathrm{Rh}-101$ & & $2.540 \mathrm{E}-04$ & $2.540 \mathrm{E}-04$ & $0.000 \mathrm{E}+00$ & 8. $000 E \div 05$ \\
\hline $\mathrm{Rh}-102 \mathrm{~m}$ & & $2.540 \mathrm{E}-04$ & $2.540 \mathrm{E}-04$ & $0.000 \mathrm{E}+00$ & $9.400 E+03$ \\
\hline Rh-102 & & $2.540 \mathrm{E}-04$ & $2.540 \mathrm{E}-04$ & $0.000 E+00$ & $1.900 E+05$ \\
\hline Se-79 & & 2. $540 \mathrm{E}-04$ & $2.540 \mathrm{E}-04$ & $0.000 E+00$ & $1.100 \mathrm{E}+02$ \\
\hline$S i-32$ & & $2.540 \mathrm{E}-04$ & $2.540 \mathrm{E}-04$ & $0.000 E+00$ & $3.400 \mathrm{E}+02$ \\
\hline $\mathrm{Sm}-146$ & & $2.540 \mathrm{E}-04$ & $2.540 \mathrm{E}-04$ & $0.000 E+00$ & $1.400 \mathrm{E}+00$ \\
\hline Sm-15I & & $2.540 \mathrm{E}-04$ & $2.540 \mathrm{E}-04$ & $0.000 E+00$ & $4.300 E+04$ \\
\hline $\mathrm{Sn}-121 \mathrm{~m}$ & & $2.540 \mathrm{E}-04$ & $2.540 \mathrm{E}-04$ & $0.000 E+00$ & $1.100 E+03$ \\
\hline Sr-90 & & $2.540 \mathrm{E}-04$ & $2.540 \mathrm{OE}-04$ & $0.000 \mathrm{E}+00$ & $1.200 \mathrm{E}+05$ \\
\hline $\mathrm{Tb}-157$ & & $2.540 \mathrm{E}-04$ & $2.540 E-04$ & $0.000 \mathrm{E}+00$ & $6.600 \mathrm{E}+05$ \\
\hline $\mathrm{Tb}-158$ & & $2.540 \mathrm{E}-04$ & $2.540 \mathrm{E}-04$ & $0.000 \mathrm{E}+00$ & $3.600 E+05$ \\
\hline TC-97 & & $2.540 E-04$ & $2.540 \mathrm{E}-04$ & $0.000 E+00$ & $1.500 \mathrm{E}+00$ \\
\hline TC-98 & & $2.540 E-04$ & $2.540 \mathrm{E}-04$ & $0.000 E+00$ & $4.800 E-01$ \\
\hline TC-99 & & $2.540 \mathrm{E}-04$ & $2.540 \mathrm{E}-04$ & $0.000 \mathrm{E}+00$ & $9.300 \mathrm{E}+01$ \\
\hline$T i-44$ & & $2.540 \mathrm{E}-04$ & $2.540 \mathrm{E}-04$ & $0.000 E+00$ & $4.000 E+05$ \\
\hline TI-204 & & $2.540 \mathrm{E}-04$ & $2.540 \mathrm{E}-04$ & $0.000 \mathrm{E}+00$ & $5.700 E+07$ \\
\hline$V-49$ & & $2.540 \mathrm{E}-04$ & $2.540 \mathrm{E}-04$ & $0.000 \mathrm{E}+00$ & $6.000 E+06$ \\
\hline W-18I & & $2.540 \mathrm{E}-04$ & $2.540 \mathrm{E}-04$ & $0.000 \mathrm{E}+00$ & $9.000 \mathrm{E}+05$ \\
\hline Zr-93 & & $2.540 \mathrm{E}-04$ & $2.540 \mathrm{E}-04$ & $0.000 E+00$ & $2.300 E+01$ \\
\hline
\end{tabular}




\begin{tabular}{|c|c|c|c|}
\hline & AQUIFER & AQUIFER & VERTICAL \\
\hline NUCLIDE & SORPTION & RETARDATION & SORPTION \\
\hline $\mathrm{Ag}-108 \mathrm{~m}$ & $9.000 E+01$ & $4.510 E+02$ & $9.000 \mathrm{E}+0 \mathrm{I}$ \\
\hline Al-26 & $1.500 E+03$ & $7.501 \mathrm{E}+03$ & $1.500 \mathrm{E}+03$ \\
\hline$A x-39$ & $0.000 E+00$ & $1.000 \mathrm{E}+00$ & $0.000 E+00$ \\
\hline$A r-42$ & $0.000 \mathrm{E}+00$ & $1.000 \mathrm{E}+00$ & $0.000 \mathrm{E}+00$ \\
\hline $\mathrm{Ba}-133$ & $5.200 \mathrm{E}+01$ & $2.610 \mathrm{E}+02$ & $5.200 E+01$ \\
\hline $\mathrm{Be}-10$ & $2.500 \mathrm{E}+02$ & 1. $251 E+03$ & $2.500 \mathrm{E}+02$ \\
\hline $\mathrm{Bi}-207$ & $1.000 \mathrm{E}+02$ & $5.010 \mathrm{E}+02$ & $1.000 \mathrm{E}+02$ \\
\hline C- 14 & $2.000 \mathrm{E}+00$ & $1.100 \mathrm{E}+01$ & $2.000 \mathrm{E}+00$ \\
\hline $\mathrm{Ca}-41$ & $5.000 E+00$ & $2.600 \mathrm{E}+01$ & $5.000 \mathrm{E}+00$ \\
\hline$c d-113 m$ & $8.000 \mathrm{E}+01$ & $4.010 \mathrm{E}+02$ & $8.000 \mathrm{E}+01$ \\
\hline Cl-36 & $1.700 \mathrm{E}+00$ & $9.500 \mathrm{E}+00$ & $1.700 \mathrm{E}+00$ \\
\hline Co-60 & $6.000 \mathrm{E}+01$ & $3.010 \mathrm{E}+02$ & $6.000 \mathrm{E}+01$ \\
\hline$C s-135$ & $3.300 \mathrm{E}+02$ & $1.651 \mathrm{E}+03$ & $3.300 \mathrm{E}+02$ \\
\hline Cs -137 & $3.300 \mathrm{E}+02$ & $1.651 \mathrm{E}+03$ & $3.300 E+02$ \\
\hline Eu-150 & $2.400 \mathrm{E}+02$ & $1.201 \mathrm{E}+03$ & $2.400 \mathrm{E}+02$ \\
\hline Eu-152 & $2.400 \mathrm{E}+02$ & $1.201 \mathrm{E}+03$ & $2.400 \mathrm{E}+02$ \\
\hline Eu-154 & $2.400 \mathrm{E}+02$ & $1.201 E+03$ & $2.400 \mathrm{E}+02$ \\
\hline Fe -60 & $2.200 \mathrm{E}+02$ & $1.101 \mathrm{E}+03$ & $2.200 \mathrm{E}+02$ \\
\hline Gd-148 & $2.400 E+02$ & $1.201 \mathrm{E}+03$ & $2.400 E+02$ \\
\hline Ge-68 & $1.300 \mathrm{E}+02$ & $6.510 \mathrm{E}+02$ & $1.500 \mathrm{E}+02$ \\
\hline $\mathrm{H}-3$ & $0.000 \mathrm{E}+00$ & $1.000 \mathrm{E}+00$ & $0.000 \mathrm{E}+00$ \\
\hline $\mathrm{H} f-178 \mathrm{~m}$ & $4.500 E+02$ & $2.251 E+03$ & $4.500 \mathrm{E}+02$ \\
\hline Hf-182 & $4.500 \mathrm{E}+02$ & $2.251 E+03$ & $4.500 \mathrm{E}+02$ \\
\hline Ho- $166 \mathrm{~m}$ & $2.500 \mathrm{E}+02$ & $1.251 \mathrm{E}+03$ & $2.500 \mathrm{E}+02$ \\
\hline$I-129$ & $6.000 \mathrm{E}-01$ & $4.000 \mathrm{E}+00$ & $1.000 \mathrm{E}+00$ \\
\hline $\mathrm{K}-40$ & $1.500 \mathrm{E}+01$ & $7.600 \mathrm{E}+01$ & $1.500 \mathrm{E}+01$ \\
\hline$K r-85$ & $0.000 \mathrm{E}+00$ & $1.000 \mathrm{E}+00$ & $0.000 \mathrm{E}+00$ \\
\hline La-137 & $1.200 \mathrm{E}+03$ & $6.001 E+03$ & $1.200 \mathrm{E}+03$ \\
\hline La- -138 & $1.200 \mathrm{E}+03$ & $6.001 \mathrm{E}+03$ & $1.200 \mathrm{E}+03$ \\
\hline$M n-53$ & $5.000 \mathrm{E}+01$ & $2.510 \mathrm{E}+02$ & $5.000 E+01$ \\
\hline Mo-93 & $1.000 E+01$ & $5.100 \mathrm{E}+01$ & $1.000 \mathrm{E}+01$ \\
\hline $\mathrm{Na}-22$ & $7.600 \mathrm{E}+01$ & $3.810 E+02$ & $7.600 \mathrm{E}+01$ \\
\hline $\mathrm{Nb}-93 \mathrm{~m}$ & $1.600 \mathrm{E}+02$ & $8.010 E+02$ & $1.600 \mathrm{E}+02$ \\
\hline $\mathrm{Nb}-94$ & $1.600 \mathrm{E}+02$ & $8.010 E+02$ & $1.600 \mathrm{E}+02$ \\
\hline$N i-59$ & $4.000 E+02$ & $2.001 E+03$ & $4.000 \mathrm{E}+02$ \\
\hline$N i-63$ & $4.000 E+02$ & $2.001 E+03$ & $4.000 \mathrm{E}+02$ \\
\hline $\mathrm{Pb}-202$ & $2.700 \mathrm{E}+02$ & $1.351 \mathrm{E}+03$ & $2.700 E+02$ \\
\hline $\mathrm{Pb}-205$ & $2.700 E+02$ & $1.351 \mathrm{E}+03$ & $2.700 \mathrm{E}+02$ \\
\hline$P m-145$ & $2.400 E \div 02$ & $1.201 E+03$ & $2.400 \mathrm{E} \div 02$ \\
\hline Pt -193 & $3.000 \mathrm{E}+01$ & $1.510 \mathrm{E}+02$ & $3.000 E+01$ \\
\hline Rh-101 & $5.200 \mathrm{E}+01$ & $2.610 \mathrm{E}+02$ & $5.200 \mathrm{E}+01$ \\
\hline $\mathrm{Rh}-102 \mathrm{~m}$ & $5.200 \mathrm{E}+01$ & $2.610 \mathrm{E}+02$ & $5.200 \mathrm{E}+01$ \\
\hline Rh-102 & $5.200 \mathrm{E}+01$ & $2.610 \mathrm{E}+02$ & $5.200 E+01$ \\
\hline Se-79 & $1.500 \mathrm{E}+02$ & $7.510 \mathrm{E}+02$ & $1.500 \mathrm{E}+02$ \\
\hline Si-32 & $3.500 \mathrm{E}+01$ & $1.760 \mathrm{E}+02$ & $3.500 E+01$ \\
\hline Sm-146 & $2.450 \mathrm{E}+02$ & $1.226 \mathrm{E}+03$ & $2.450 \mathrm{E}+02$ \\
\hline Sm-151 & $2.450 E+02$ & $1.226 \mathrm{E}+03$ & $2.450 \mathrm{E} \div 02$ \\
\hline $\mathrm{Sn}-121 \mathrm{~m}$ & $1.300 E+02$ & $6.510 \mathrm{E}+02$ & $1.300 \mathrm{E}+02$ \\
\hline SI -90 & $1.000 E \div 01$ & $5.100 \mathrm{E}+01$ & $1.000 E+01$ \\
\hline$T b-157$ & $2.400 E+02$ & $1.201 \mathrm{E}+03$ & $2.400 \mathrm{E}+02$ \\
\hline $\mathrm{Tb}-158$ & $2.400 \mathrm{E}+02$ & $1.201 \mathrm{E}+03$ & $2.400 \mathrm{E}+02$ \\
\hline TC-97 & $3.600 \mathrm{E}-01$ & $2.800 \mathrm{E}+00$ & $3.600 \mathrm{E}-01$ \\
\hline Tc-98 & $3.600 \mathrm{E}-01$ & $2.800 \mathrm{E}+00$ & $3.600 \mathrm{E}-01$ \\
\hline TC-99 & $3.600 \mathrm{E}-01$ & $2.800 \mathrm{E}+00$ & $3.600 E-01$ \\
\hline$T i-44$ & $3.900 \mathrm{E}+02$ & $1.951 \mathrm{E}+03$ & $3.900 \mathrm{E}+02$ \\
\hline Tl-204 & $6.000 \mathrm{E}+02$ & $3.001 \mathrm{E}+03$ & $6.000 \mathrm{E}+02$ \\
\hline$V-49$ & $1.600 \mathrm{E}+02$ & $8.010 \mathrm{E}+02$ & $1.600 \mathrm{E}+02$ \\
\hline W-181 & $1.000 \mathrm{E}+02$ & $5.010 \mathrm{E}+02$ & $1.000 \mathrm{E}+02$ \\
\hline Zr-93 & $6.000 \mathrm{E}+02$ & $3.001 \mathrm{E}+03$ & $6.000 \mathrm{E}+02$ \\
\hline
\end{tabular}

VERTICAL RETARDATION

$9.010 \mathrm{~d}+02$

$1.500 \mathrm{E}+04$

$1.000 \mathrm{E}+00$

$1.000 \mathrm{E}+00$

5. $210 \mathrm{E}+02$

$2.501 E+03$

I. $001 \mathrm{E}+03$

2.100E+01

$5.100 \mathrm{E}+01$

$8.010 \mathrm{E}+02$

$1.800 \mathrm{E}+01$

$6.010 \mathrm{E}+02$

$3.301 \mathrm{E}+03$

$3.301 \mathrm{E}+03$

$2.401 E+03$

$2.401 E+03$

$2.401 \mathrm{E}+03$

$2.201 E+03$

$2.401 \mathrm{E}+03$

I. $501 \mathrm{E}+03$

$1.000 \mathrm{E}+00$

$4.501 \mathrm{E}+03$

$4.501 \mathrm{E}+03$

$2.501 \mathrm{E}+03$

1. $100 \mathrm{E}+01$

$1.510 \mathrm{E}+02$

$1.000 \mathrm{E}+00$

$1.200 \mathrm{E}+04$

1. $200 \mathrm{E}+04$

$5.010 \mathrm{E}+02$

$1.010 \mathrm{E}+02$

$7.610 \mathrm{E}+02$

$1.601 \mathrm{E}+03$

$1.601 E+03$

4. $001 E+03$

$4.001 E+03$

2. $701 E+03$

2. $701 E+03$

2. $401 \mathrm{E}+03$

$3.010 \mathrm{E}+02$

5. $210 \mathrm{E}+02$

5. $210 \mathrm{E}+02$

5. $210 \mathrm{E}+02$

1. $501 \mathrm{E}+03$

$3.510 \mathrm{E}+02$

$2.451 E+03$

$2.451 \mathrm{E}+03$

$1.301 \mathrm{E}+03$

1. $010 \mathrm{E}+02$

$2.401 E+03$

$2.401 \mathrm{E}+03$

$4.600 \mathrm{E}+00$

$4.600 \mathrm{E}+00$

$4.600 \mathrm{E}+00$

$3.901 \mathrm{E}+03$

$6.001 \mathrm{E}+03$

$1.601 \mathrm{E}+03$

$1.001 \mathrm{E}+03$

$6.001 E+03$ 
BIOACCUMULATION FACTORS

\begin{tabular}{|c|c|c|c|c|}
\hline & SOIL-PI_ANT & SOIL-PLANT & FORAGE - MILK & FORAGE-MEAI \\
\hline NUCLIDE & $\mathrm{BV}$ & $\mathrm{Br}$ & $\mathrm{Fm}(\mathrm{D} / \mathrm{L})$ & Ff $\quad(D / K G)$ \\
\hline $\mathrm{Ag}-108 \mathrm{~m}$ & $1.500 \mathrm{E}-01$ & $1.500 \mathrm{E}-02$ & $5.000 \mathrm{E}-02$ & $1.700 \mathrm{E}-02$ \\
\hline Al-26 & $6.600 \mathrm{E}-04$ & $6.600 \mathrm{E}-05$ & $1.200 \mathrm{E}-03$ & $4.000 \mathrm{E}-02$ \\
\hline$A x-39$ & $6.000 E-01$ & $6.000 \mathrm{E}-02$ & $2.000 \mathrm{E}-02$ & $2.000 \mathrm{E}-02$ \\
\hline$A x-42$ & $6.000 \mathrm{E}-01$ & $6.000 \mathrm{E}-02$ & $2.000 \mathrm{E}-02$ & $2.000 \mathrm{E}-02$ \\
\hline $\mathrm{Ba}-133$ & $5.000 \mathrm{E}-03$ & $5.000 \mathrm{E}-04$ & $4.000 \mathrm{E}-04$ & $3.200 \mathrm{E}-03$ \\
\hline $\mathrm{Be}-10$ & $4.200 E-04$ & $4.200 \mathrm{E}-05$ & $1.000 \mathrm{E}-04$ & $1.000 \mathrm{E}-03$ \\
\hline $\mathrm{Bi}-207$ & $1.500 \mathrm{E}-01$ & $1.500 \mathrm{E}-02$ & $5.000 \mathrm{E}-04$ & $1.300 \mathrm{E}-02$ \\
\hline C-14 & $5.500 \mathrm{E}+00$ & $5.500 \mathrm{E}-01$ & $1.200 \mathrm{E}-02$ & $3.100 \mathrm{E}-02$ \\
\hline $\mathrm{Ca}-41$ & $3.600 \mathrm{E}-02$ & $3.600 \mathrm{E}-03$ & $8.000 \mathrm{E}-03$ & $4.000 \mathrm{E}-03$ \\
\hline $\mathrm{cd}-113 \mathrm{~m}$ & $3.000 \mathrm{E}-01$ & $3.000 \mathrm{E}-02$ & $1.200 \mathrm{E}-04$ & $5.300 E-04$ \\
\hline Cl-36 & $5.000 \mathrm{E}+00$ & $5.000 \mathrm{E}-0 \mathrm{I}$ & $5.000 \mathrm{E}-02$ & $8.000 \mathrm{E}-02$ \\
\hline Co-60 & $9.400 \mathrm{E}-03$ & $9.400 \mathrm{E}-04$ & $1.000 \mathrm{E}-03$ & $1.300 \mathrm{E}-02$ \\
\hline $\mathrm{Cs}-135$ & 1.000 E-02 & $1.000 \mathrm{E}-03$ & $1.200 \mathrm{E}-02$ & $4.000 E-03$ \\
\hline $\mathrm{Cs}-137$ & $1.000 \mathrm{E}-02$ & $1.000 \mathrm{E}-03$ & $1.200 \mathrm{E}-02$ & $4.000 E-03$ \\
\hline Eu-150 & $2.500 E-03$ & $2.500 \mathrm{E}-04$ & $5.000 E-06$ & $4.800 E-03$ \\
\hline$E u-152$ & $2.500 E-03$ & $2.500 \mathrm{E}-04$ & $5.000 \mathrm{E}-06$ & $4.800 E-03$ \\
\hline Eu-154 & $2.500 E-03$ & $2.500 \mathrm{E}-04$ & $5.000 \mathrm{E}-06$ & $4.800 E-03$ \\
\hline $\mathrm{Fe}-60$ & $6.600 \mathrm{E}-04$ & $6.600 \mathrm{E}-05$ & $1.200 \mathrm{E}-03$ & $4.000 \mathrm{E}-02$ \\
\hline Gd-148 & $2.600 \mathrm{E}-03$ & $2.600 \mathrm{E}-04$ & $5.000 \mathrm{E}-06$ & $3.400 \mathrm{E}-03$ \\
\hline $\mathrm{Ge}-68$ & $2.500 \mathrm{E}-03$ & $2.500 \mathrm{E}-04$ & $2.500 \mathrm{E}-03$ & $8.000 \mathrm{E}-02$ \\
\hline $\mathrm{H}-3$ & $4.800 \mathrm{E}+00$ & $4.800 \mathrm{E}-01$ & 1.000E-02 & $1.200 \mathrm{E}-02$ \\
\hline $\mathrm{Hf}-178 \mathrm{~m}$ & $1.700 \mathrm{E}-04$ & $1.700 \mathrm{E}-05$ & $5.000 \mathrm{E}-06$ & $4.000 E-01$ \\
\hline $\mathrm{HE}-182$ & $1.700 \mathrm{E}-04$ & $1.700 \mathrm{E}-05$ & $5.000 \mathrm{E}-06$ & $4.000 \mathrm{E}-01$ \\
\hline Ho- $166 \mathrm{~m}$ & $2.600 \mathrm{E}-03$ & $2.600 \mathrm{E}-04$ & $5.000 \mathrm{E}-06$ & $4.400 \mathrm{E}-03$ \\
\hline$I-129$ & $2.000 \mathrm{E}-02$ & $2.000 \mathrm{E}-03$ & $6.000 E-03$ & $2.900 E-03$ \\
\hline$K-40$ & $3.700 \mathrm{E}-01$ & $3.700 \mathrm{E}-02$ & $1.000 \mathrm{E}-02$ & $1.200 \mathrm{E}-02$ \\
\hline$K x-85$ & $3.000 \mathrm{E}+00$ & $3.000 \mathrm{E}-01$ & $2.000 \mathrm{E}-02$ & $2.000 E-02$ \\
\hline $\mathrm{La}-137$ & $2.500 E-03$ & $2.500 E-04$ & $5.000 \mathrm{E}-06$ & $2.000 \mathrm{E}-04$ \\
\hline$L a-138$ & $2.500 \mathrm{E}-03$ & $2.500 \mathrm{E}-04$ & $5.000 \mathrm{E}-06$ & $2.000 E-04$ \\
\hline $\mathrm{Mn}-53$ & $2.900 \mathrm{E}-02$ & $2.900 \mathrm{E}-03$ & $2.500 E-04$ & $8.000 E-04$ \\
\hline Mo-93 & $1.200 \mathrm{E}-01$ & $1.200 \mathrm{E}-02$ & $7.500 \mathrm{E}-03$ & $8.000 E-03$ \\
\hline $\mathrm{Na}-22$ & $5.200 \mathrm{E}-02$ & $5.200 \mathrm{E}-03$ & $4.000 \mathrm{E}-02$ & $3.000 \mathrm{E}-02$ \\
\hline $\mathrm{Nb}-93 \mathrm{~m}$ & $9.400 \mathrm{E}-03$ & $9.400 \mathrm{E}-04$ & $2.500 \mathrm{E}-03$ & $2.800 E-01$ \\
\hline $\mathrm{Nb}-94$ & $9.400 \mathrm{E}-03$ & $9.400 \mathrm{E}-04$ & $2.500 \mathrm{E}-03$ & $2.800 \mathrm{E}-01$ \\
\hline $\mathrm{Ni}-59$ & $1.900 \mathrm{E}-02$ & $1.900 \mathrm{E}-03$ & $6.700 \mathrm{E}-03$ & $5.300 \mathrm{E}-03$ \\
\hline $\mathrm{Ni}-63$ & $1.900 \mathrm{E}-02$ & $1.900 \mathrm{E}-03$ & $6.700 \mathrm{E}-03$ & $5.300 \mathrm{E}-03$ \\
\hline $\mathrm{Pb}-202$ & $6.800 \mathrm{E}-02$ & $6.800 \mathrm{E}-03$ & $6.200 \mathrm{E}-04$ & $2.900 \mathrm{E}-04$ \\
\hline $\mathrm{Pb}-205$ & $6.800 \mathrm{E}-02$ & $6.800 \mathrm{E}-03$ & $6.200 \mathrm{E}-04$ & $2.900 \mathrm{E}-04$ \\
\hline $\mathrm{Pm}-145$ & $2.500 \mathrm{E}-03$ & $2.500 \mathrm{E}-04$ & $5.000 \mathrm{E}-06$ & $4.800 \mathrm{E}-03$ \\
\hline pt -193 & $1.300 \mathrm{E}+01$ & $1.300 E+00$ & $5.000 E-03$ & $1.500 \mathrm{E}-03$ \\
\hline Rh-101 & $1.300 \mathrm{E}+01$ & $1.300 \mathrm{E}+00$ & $1.000 \mathrm{E}-02$ & $1.500 \mathrm{E}-03$ \\
\hline$R h-102 m$ & $1.300 E+01$ & $1.300 \mathrm{E}+00$ & $1.000 \mathrm{E}-02$ & $1.500 \mathrm{E}-03$ \\
\hline Rh-102 & $1.300 \mathrm{E}+01$ & $1.300 E+00$ & $1.000 \mathrm{E}-02$ & $1.500 \mathrm{E}-03$ \\
\hline Se-79 & $1.300 \mathrm{E}+00$ & $1.300 \mathrm{E}-01$ & $4.500 \mathrm{E}-02$ & $1.500 \mathrm{E}-02$ \\
\hline Si-32 & $5.500 E+00$ & $5.500 \mathrm{E}-01$ & $1.200 E-02$ & $3.100 \mathrm{E}-02$ \\
\hline $\mathrm{Sm}-146$ & $2.500 \mathrm{E}-03$ & $2.500 \mathrm{E}-04$ & $5.000 \mathrm{E}-06$ & $5.000 \mathrm{E}-03$ \\
\hline Sm-151 & $2.500 E-03$ & $2.500 \mathrm{E}-04$ & $5.000 \mathrm{E}-06$ & $5.000 E-03$ \\
\hline Sn-121m & $2.500 \mathrm{E}-03$ & $2.500 \mathrm{E}-04$ & $2.500 \mathrm{E}-03$ & $8.000 \mathrm{E}-02$ \\
\hline Sr-90 & $1.700 \mathrm{E}-02$ & $1.700 \mathrm{E}-03$ & $8.000 E-04$ & $6.000 \mathrm{E}-04$ \\
\hline $\mathrm{Tb}-157$ & $2.600 \mathrm{E}-03$ & $2.600 \mathrm{E}-04$ & $5.000 E-06$ & $3.400 \mathrm{E}-03$ \\
\hline $\mathrm{Tb}-158$ & $2.600 E-03$ & $2.600 \mathrm{E}-04$ & $5.000 \mathrm{E}-06$ & $3.400 \mathrm{E}-03$ \\
\hline Tc-97 & $2.500 E-01$ & $2.500 \mathrm{E}-02$ & $2.500 \mathrm{E}-02$ & $4.000 \mathrm{E}-01$ \\
\hline TC-98 & $2.500 \mathrm{E}-01$ & $2.500 E-02$ & $2.500 E-02$ & $4.000 \mathrm{E}-01$ \\
\hline TC-99 & $2.500 \mathrm{E}-01$ & $2.500 \mathrm{E}-02$ & $2.500 \mathrm{E}-02$ & $4.000 E-01$ \\
\hline$T i-44$ & $2.500 E-04$ & $2.500 E-05$ & $2.200 \mathrm{E}-03$ & $2.400 E-03$ \\
\hline Tl-204 & $2.500 \mathrm{E}-01$ & $2.500 \mathrm{E}-02$ & $2.200 \mathrm{E}-02$ & $4.000 E-02$ \\
\hline$v-49$ & $2.500 \mathrm{E}-04$ & $2.500 E-05$ & $2.200 E-03$ & $2.400 E-03$ \\
\hline$W-181$ & $1.200 \mathrm{E}-01$ & $1.200 \mathrm{E}-02$ & $7.500 \mathrm{E}-03$ & $8.000 E-03$ \\
\hline$z x-93$ & $1.700 \mathrm{E}-04$ & $1.700 \mathrm{E}-05$ & $5.000 \mathrm{E}-06$ & $3.400 \mathrm{E}-02$ \\
\hline
\end{tabular}




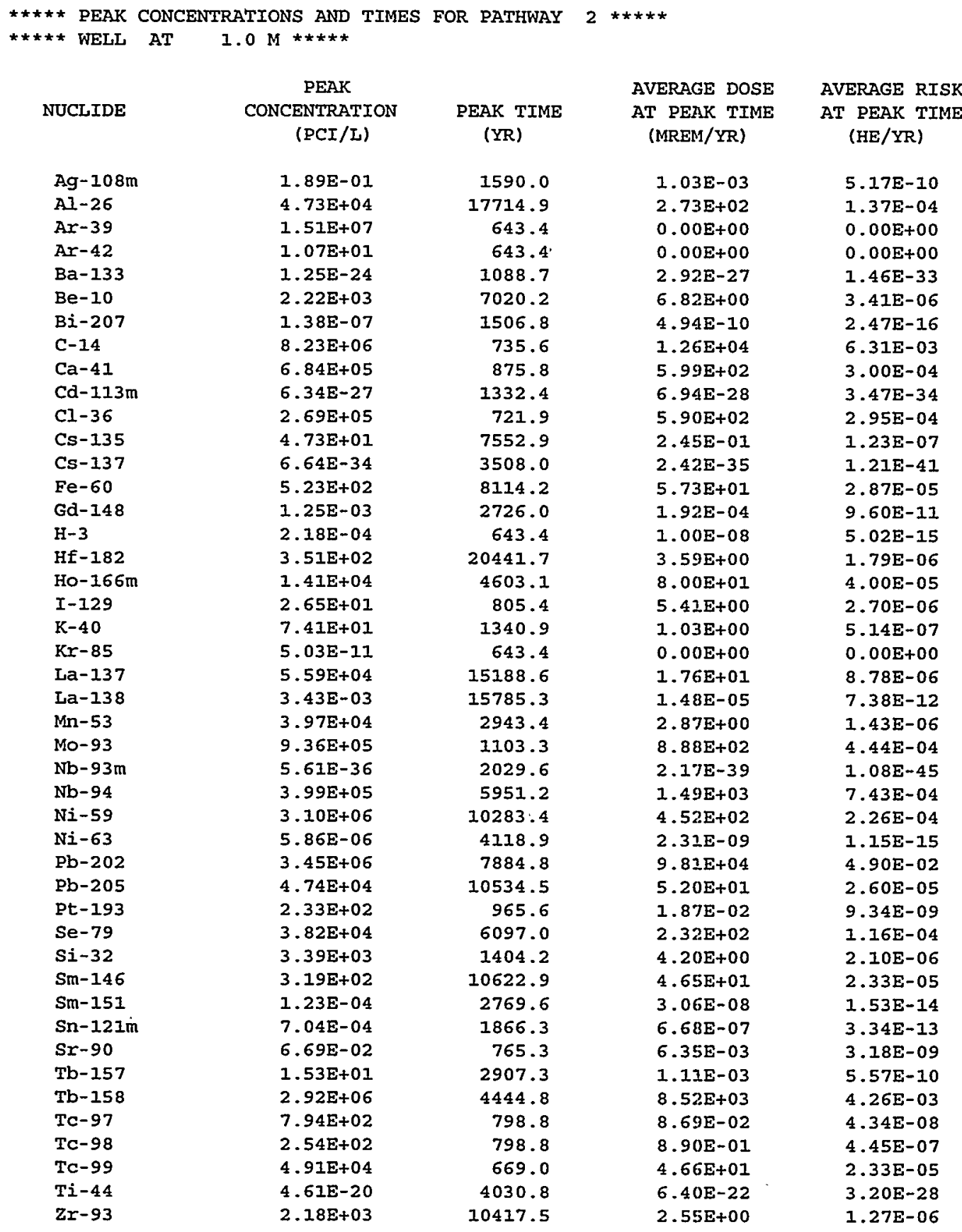




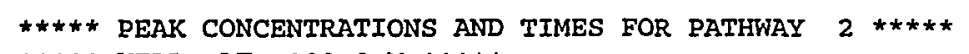

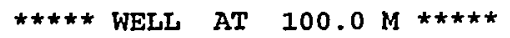

\begin{tabular}{|c|c|c|c|c|c|}
\hline & PEAK & & AVERAGE DOSE & AVERAGE RISK & \\
\hline NUCLIDE & $\begin{array}{c}\text { CONCENTRATION } \\
\text { (PCI/I) }\end{array}$ & $\begin{array}{l}\text { PEAK TIME } \\
\text { (YR) }\end{array}$ & $\begin{array}{l}\text { AT PEAK TIME } \\
\text { (MREM/YR) }\end{array}$ & $\begin{array}{c}\text { AT PEAK TIME } \\
(\mathrm{HE} / \mathrm{YR})\end{array}$ & \\
\hline $\mathrm{Ag}-1.08 \mathrm{~m}$ & $4.72 E-12$ & 6059.3 & $2.59 \mathrm{E}-14$ & $1.29 \mathrm{E}-20$ & \\
\hline Al-26 & $4.41 E+04$ & 92294.7 & $2.54 \mathrm{E}+02$ & $1.27 \mathrm{E}-04$ & \\
\hline Ar-39 & $1.47 \mathrm{E}+07$ & 653.3 & $0.00 E+00$ & $0.00 E+00$ & \\
\hline Ar -42 & $8.71 E+00$ & 653.3 & $0.00 E+00$ & $0.00 \mathrm{E}+00$ & \\
\hline$B e-10$ & $2.20 E+03$ & 20409.2 & $6.75 E+00$ & $3.38 \mathrm{E}-06$ & \\
\hline$C-14$ & $8.12 E+06$ & 844.5 & $1.25 E+04$ & $6.23 E-03$ & \\
\hline $\mathrm{Ca}-41$ & $6.83 E+05$ & 1134.4 & $5.98 E+02$ & $2.99 \mathrm{E}-04$ & \\
\hline$C l-36$ & $2.69 E+05$ & 826.2 & $5.90 E+02$ & $2.95 \mathrm{E}-04$ & \\
\hline $\mathrm{Cs}-135$ & $4.69 E+01$ & 29681.0 & $2.43 E-01$ & $1.21 \mathrm{E}-07$ & \\
\hline $\mathrm{Fe}-60$ & 5. IIE+02 & 17688.6 & $5.60 \mathrm{E}+01$ & $2.80 E-05$ & \\
\hline $\mathrm{H}-3$ & $1.25 E-04$ & 653.3 & $5.74 E-09$ & $2.87 \mathrm{E}-15$ & \\
\hline $\mathrm{HE}-182$ & $3.48 E+02$ & 32934.1 & $3.56 E+00$ & $1.78 \mathrm{E}-06$ & \\
\hline HO- $166 \mathrm{~m}$ & $1.09 E+01$ & 16996.2 & $6.20 E-02$ & $3.10 E-08$ & \\
\hline$I-129$ & $2.65 \mathrm{E}+01$ & 854.9 & $5.41 \mathrm{E}+00$ & $2.70 \mathrm{E}-06$ & \\
\hline$K-40$ & $7.41 E+01$ & 2278.0 & $1.03 E+00$ & $5.14 E-07$ & \\
\hline$K r-85$ & $2.65 \mathrm{E}-11$ & 653.3 & $0.00 E+00$ & $0.00 \mathrm{E}+00$ & \\
\hline La-137 & $2.77 E+04$ & 75589.0 & $8.69 E+00$ & $4.34 E-06$ & \\
\hline La-138 & $3.38 E-03$ & 113396.5 & $1.46 \mathrm{E}-05$ & $7.29 \mathrm{E}-12$ & \\
\hline$M n-53$ & $3.96 E+04$ & 5555.0 & $2.87 \mathrm{E}+00$ & $1.43 \mathrm{E}-06$ & \\
\hline MO-93 & $8.46 \mathrm{E}+05$ & 1609.0 & $8.03 E+02$ & $4.02 E-04$ & - \\
\hline $\mathrm{Nb}-94$ & $3.03 E+05$ & 13936.7 & $1.13 E+03$ & $5.64 \mathrm{E}-04$ & \\
\hline$N i-59$ & $2.60 \mathrm{E}+06$ & 27837.9 & $3.79 \mathrm{E}+02$ & $1.90 \mathrm{E}-04$ & \\
\hline $\mathrm{Pb}-202$ & $3.34 \mathrm{E}+06$ & 21277.7 & $9.50 \mathrm{E}+04$ & $4.75 E-02$ & • \\
\hline $\mathrm{Pb}-205$ & $4.74 \mathrm{E}+04$ & 25017.9 & 5.19E+01 & $2.59 \mathrm{E}-05$ & \\
\hline$p t-193$ & $2.25 E-07$ & 2461.9 & I. $80 \mathrm{E}-11$ & $9.02 E-18$ & \\
\hline Se-79 & $3.53 \mathrm{E}+04$ & 13403.2 & $2.14 \mathrm{E}+02$ & $1.07 E-04$ & \\
\hline$S i-32$ & $8.68 E+01$ & 3147.4 & $1.08 \mathrm{E}-01$ & $5.39 E-08$ & \\
\hline $\mathrm{Sm}-146$ & 3.18E+02 & 19724.6 & $4.65 \mathrm{E}+01$ & $2.32 E-05$ & \\
\hline$S n-121 m$ & $1.88 E-29$ & 8317.1 & $1.79 E-32$ & $8.94 E-39$ & \\
\hline$S r-90$ & $3.20 E-07$ & 1266.8 & $3.04 \mathbf{E}-08$ & $1.52 \mathrm{E}-14$ & \\
\hline $\mathrm{Tb}-157$ & $1.90 \mathrm{E}-23$ & 14809.2 & $1.39 \mathrm{E}-27$ & $6.94 \mathrm{E}-34$ & \\
\hline $\mathrm{Tb}-158$ & $3.01 \mathrm{E}+03$ & 16253.8 & $8.79 \mathrm{E}+00$ & $4.39 E-06$ & \\
\hline TC-97 & $7.94 E+02$ & 833.5 & $8.69 E-02$ & $4.34 E-08$ & \\
\hline Tc-98 & $2.54 E+02$ & 833.5 & $8.90 \mathrm{E}-01$ & $4.45 \mathrm{E}-07$ & $\cdot$ \\
\hline TC-99 & $4.91 \mathrm{E}+04$ & 698.0 & $4.66 \mathrm{E}+02$ & $2.33 E-05$ & \\
\hline Zr-93 & $2.13 E+03$ & 39890.6 & $2.48 \mathrm{E}+00$ & $1.24 E-06$ & \\
\hline
\end{tabular}


GROUNDWATER PATHWAY SCOPING ANALYSIS

FOR DISPOSAL OF APT AND TEF WASTES

WSRC-RP-98-00729

Distribution:

W. E. Stevens, 773-A

J. R. Cook, 773-43A

E. L. Wilhite, 773-A

T. Hang, 773-42A

L. B. Collard, 773-43A

V. J. Reymers, 773-43A

R.A. Hane, 773-43A

D.E. Grove, $730-1 B$

S.S. Patel, 730-1B

G.T. Chandler, 773-A

H. Shih, Burns \& Roe

G. DeCamp, Burns \& Roe

K. Barbour, Burns \& Roe

J.L. Anderson, LANL
W. T. Goldston, 705-3C

M. J. Ades, 705-3C

D. F. Sink, 724-15E

L.C. Thomas, 705-3C

M.W. Lewis, 724-7E

R.M. Seaborn, 705-3C

P.I. Hudson, 705-3C

G.R. Vozniak, 730-1B

J.R. Purucker, 730-1B

E.A. Clark, 773-A

P.L. Nowacki, 730-1B

A. Ugelow, Burns \& Roe

A. Coons, Burns \& Roe
R. W. Reynolds, 703-45A

J. L. England, LANL

S. O. Sheetz, LANL

R. H. Hsu, 773-A

R. A. Smith, 730-1B

J.S. Roberts, 703-45A

F.H. Brown, 730-1B

W.F. Brizes, 730-1B

D. Morris, Burns \& Roe

C. Drewry, Burns \& Roe

P. Lanik, Burns \& Roe

H. Cohen, Burns \& Roe

M. Richards, GA-LANL 